\title{
A distributed dopamine-gated circuit underpins reproductive state-dependent behavior in
} Drosophila females

Ariane C. Boehm ${ }^{\mathrm{a}, \mathrm{b}}$, Anja B. Friedrich ${ }^{\mathrm{a}}$, Paul Bandow ${ }^{\mathrm{a}, \mathrm{b}}$, K.P. Siju ${ }^{\mathrm{a}}$, Sydney Hunt ${ }^{\mathrm{a}}$, Julia Claussen ${ }^{\mathrm{a}}$, MarieHelen Link ${ }^{\mathrm{a}}$, Thomas F. Hofmann ${ }^{\mathrm{c}, \mathrm{d}}$, Corinna Dawid ${ }^{\mathrm{c}, \mathrm{d}}$, Ilona C. Grunwald Kadow ${ }^{\mathrm{a}, \mathrm{b}, \mathrm{c}}$

a Technical University Munich, School of Life Sciences, Neuronal Control of Metabolism,

Freising, Germany

b Graduate School of Systemic Neurosciences, Ludwig Maximilian University, Martinsried, Germany

10 c ZIEL - Institute for Food and Health, Technical University Munich, School of Life Sciences, Freising, Germany

d Technical University Munich, School of Life Sciences, Chair of Food Chemistry and Molecular Sensory Science, Freising, Germany

Corresponding author: Ilona C. Grunwald Kadow, ilona.grunwald@tum.de

\section{ABSTRACT}

Motherhood induces a drastic, sometimes long-lasting, change in internal state and behavior in most female animals. Here, we show that a mating-induced increase in olfactory attraction of female Drosophila flies to nutrients relies on interconnected neural pathways in the two higher olfactory brain regions, the lateral horn ( $\mathrm{LH}$ ) and the mushroom body (MB). Using whole brain calcium imaging, we find that mating does not induce a global change in the activity of the whole brain nor of entire brain regions, suggesting specific neuronal or network changes in the olfactory system. Systematic behavioral screening and electron microscopy (EM) connectomics identify two types of LH output neurons required for the attraction of females to polyamines -one of them previously implicated in the processing of male pheromones. In addition, we characterize multiple MB pathways capable of inducing or suppressing polyamine attraction, with synaptic connections to the identified LH neurons and a prominent role for the $\beta$ ' 1 compartment. Moreover, $\beta$ ' 1 dopaminergic neurons are modulated by mating and are sufficient to replace mating experience in virgins inducing the lasting behavioral switch in female preference. Taken together, our data in the fly suggests that reproductive state-dependent expression of female choice behavior is regulated by a dopamine-gated distributed learning circuit comprising both higher olfactory brain centers.

Keywords:

35 Female behavior, reproductive state, lateral horn, mushroom body, modulation, polyamine, Drosophila melanogaster

\section{INTRODUCTION}

External stimuli and internal states guide decisions by influencing interpretation of the environment. These decisions not only impact on the individual itself but may have severe effects on its offspring. A female animal, therefore, adapts her choices to her reproductive state. This includes not only the decision to mate, but also a preference for certain, nutrient-rich food sources or, in case of egg-laying animals, oviposition sites (Chapman and Wolfner, 2017; Sayin et al., 2018). Choices pertaining to food, nutrients or egg-laying sites frequently depend on chemosensory cues such as odors or tastes. In line with this, changes in olfactory and gustatory perception have been reported for pregnant women (Ochsenbein-Kolble et al., 2007). The 
cellular and neural mechanisms responsible for such reproductive state-dependent preferences remain incompletely understood.

A powerful mechanism for adapting preference for an odor or taste according to internal state acts within the very sensory neurons that detect the chemical (Leinwand and Chalasani, 2011). For instance, hunger increases the sensitivity of sweet taste and quenches that of bitter taste neurons in different animal species including humans (Carnell et al., 2012; Chu et al., 2014; LeDue et al., 2016; Palouzier-Paulignan et al., 2012; Rolls, 2007). The estrous cycle influences how a female mouse perceives a putative mate (Dey et al., 2015). Outside of estrus, the hormone progesterone strongly inhibits the female's male pheromone-sensitive olfactory sensory neurons (OSNs), and thereby, completely blunts her interest in males. Moreover, previous results using volume measurements in human females before, during and after pregnancy suggested that pregnancy induces long-lasting, global changes in grey matter volume in several brain regions (Hoekzema et al., 2017), indicative of modulation in multiple higher brain regions. In the genetically tractable insect, Drosophila melanogaster, reproductive state also induces dramatic shifts in the female's chemosensory perception and choices (Gou et al., 2014; Hussain et al., 2016a; Ribeiro and Dickson, 2010; Walker et al., 2015). We have previously shown that a mating-induced transient neuropeptidergic modulation of OSNs strongly increases a female fly's preference for higher concentrations of the important nutrient polyamine (Hussain et al., 2016a; Hussain et al., 2016b). This rather short-lasting sensory modulation $(\sim 24 \mathrm{~h}$ ) leads to a longer lasting change in the female's choice behavior, indicating that mating might modulate higher chemosensory processing in the female's central brain. Here, we aimed at investigating the nature of such modulations, whether they affect brain state more globally as indicated by the above-mentioned study in pregnant women (Hoekzema et al., 2017), rely on individual neurons and small circuit motifs, or whether they entail distributed neural networks across multiple brain regions.

Reproductive state-dependent behavior of flies to polyamines is well suited to address this question. Polyamines, namely putrescine, spermine and spermidine, play essential and conserved roles in most eukaryotic cells and organisms, ranging from DNA replication, cell proliferation, embryonic development to healthy aging (Miller-Fleming et al., 2015). More importantly, olfactory detection of polyamines is relatively well characterized at the sensory neuron level and depends on two co-expressed ionotropic receptors (IR), IR76b and IR41a on the fly's antenna (Hussain et al., 2016b). Within the first several hours after mating, the expression of the neuropeptide receptor, sex peptide receptor (SPR) increases by tenfold in the OSNs leading, when bound to its ligand myoinhibitory peptide (MIP), to a depression of polyamine OSN presynaptic output to the second order neurons of the olfactory system (Hussain et al., 2016a). While virgin flies show a high attraction toward low concentration of polyamines, mated flies are attracted to higher concentrations (Hussain et al., 2016a).

Attraction to nutrients, egg-laying or nest building sites are considered innate behaviors in most female animals (Cury et al., 2019). Innate olfactory behavior is thought to rely on stereotyped circuits connecting specific glomeruli in the antennal lobe (AL) to specific neurons in the lateral horn (LH) (Chin et al., 2018; Ebrahim et al., 2015; Min et al., 2013). In line with this, previous work in the fly has implicated the LH in the control of reproductive behaviors, such as responses to sex pheromones (Jefferis et al., 2007; Ruta et al., 2010). Neurons innervating or providing output of the LH show largely stereotypic responses to odors (Frechter et al., 2019; Jeanne et al., 2018). Moreover, recent studies implicated neurons in the LH in innate valence decisions and odor classification (Dolan et al., 2019; Jeanne et al., 2018; Strutz et al., 2014). Interestingly, modulation of innate behavior, for instance in the context of hunger, also involves the second higher olfactory brain center, the mushroom body (MB), a well-characterized brain region known for its role 
in associative memory formation (Bracker et al., 2013; Cohn et al., 2015; Grunwald Kadow, 2018; Heisenberg, 2003; Krashes et al., 2009; Lewis et al., 2015; Sayin et al., 2018; Tsao et al., 2018). In reproductive behavior, dopaminergic neurons (DANs) innervating the MB regulate the mated female's decision of where to lay her eggs (Azanchi et al., 2013). Moreover, DAN odor responses are modulated by the mating state of the female (Siju et al., 2020). In addition, it is worth noting that a crucial role for dopamine and the MB have been shown in the mating induced changes in male courtship learning (Keleman et al., 2012; Siegel and Hall, 1979).

Recent connectomics data has highlighted the fact that (fly) brains are made up of highly interconnected, brain-wide, recurrent neural networks consisting of tens to hundreds of neurons and of magnitudes more synapses (Li et al., 2020; Zheng et al., 2018). The mentioned examples and other work have described mostly stereotyped and/or small circuit motifs regulating mating state-dependent chemosensory behavior. How such small motifs and their role in regulating behavior can be reconciled with the brain-wide, interconnected networks seen in the fly brain remains an open question. Here, we have investigated the distributed neural circuits underpinning reproductive state-dependent female attraction to polyamines using brain-wide imaging, connectomics and behavioral screening. Our combined data suggest that a change in reproductive state modulates innate female behavior through widespread interconnected neural pathways spanning multiple brain regions.

\section{RESULTS}

\section{Specific lateral horn output neurons mediate attraction to polyamines}

Mated female flies show an increased attraction to polyamine odors, such as putrescine, as compared to virgin females due to SPR-mediated neuromodulation of polyamine sensitive OSNs (Hussain et al., 2016a). The increased attraction, interestingly, lasts for at least 9 days after mating (Fig. 1A, S1A), while the

115 neuromodulation of the OSNs appears to decline already several hours after mating (Hussain et al., 2016a). We wondered whether mating would induce other changes in other parts of the nervous system that would explain the lasting change in behavior. For example, changes in internal states might induce global brain state changes in the entire brain or whole brain regions and thereby impact multiple or even all behavior of the animal. Moreover, hormonal signaling triggers circuit maturation in Drosophila flies during the first

\section{0 week of life (Leinwand and Scott, 2021). However, mating did not appear to change brain activity globally} as in vivo whole brain lightfield imaging of 1-week old mated and virgin females stimulated with putrescine did not uncover any significant brain-wide changes (Fig. S1B,C). Similarly, we did not detect any significant differences between mated and virgin flies by analyzing separate brain regions at the spatial resolution of the lightfield microscope (Fig. S1D,E). These data suggested that mating does not induce a 125 strong global change in brain state (e.g. general arousal state), but possibly relies on modulation of specific, and possibly many, neurons and neural circuit elements relevant to a specific task.

Thus, we sought to identify the underpinning neural circuits, downstream of the OSNs, mediating this statedependent attraction to polyamines. To this end, we initially used the trans-synaptic tracing method TransTANGO (Talay et al., 2017) to identify the projection neurons (PNs) projecting from the antennal lobe (AL) 130 glomerulus (VC5), where it receives input by the OSNs detecting polyamine odors (i.e. IR41a/IR76b OSNs) to the higher brain centers. These data suggested that VC5-innervating PNs project to the $\mathrm{LH}$ and the MB calyx (Fig. S1F-G'). Given the previously reported role of the LH in odor attraction and reproductive behavior, we first focused on neurons in this brain structure. As the number of LH output neurons (LHONs) is large, we next used lightfield imaging to narrow down the putative anatomy of candidate neurons (Fig. 
GCaMP fluorescence mainly in the ventral, but also in a smaller area in the dorsal, region of the LH (Fig. 1B). By contrast, stimulation with 1\% vinegar activated primarily neurons in the dorsal part of the LH (Fig. 1B) as previously observed (Strutz et al., 2014). This suggested that putative polyamine-responding LHONs strongly innervate the ventral portion of the LH. In a complementary approach, we used the recently released online platform 'neuPrint' to search for LHNs receiving input from VC5 PNs in the electron microscope connectome of a female hemibrain (Fig. 1C, S1H) (Li et al., 2020; Scheffer et al., 2020). According to neuPrint, three different types of PNs receive direct input from the VC5 glomerulus, adPN, lvPN, and 12PN. Only one of these types, adPN, innervates both the LH and the MB calyx matching the trans-TANGO data. We again used neuPrint to identify the main LHONs innervated by adPNs. The primary LHON type receiving adPN input is AD1b2 (Fig. S1H). Most AD1b2 dendrites are indeed located in the ventral part of the LH (Fig. 1F). To test the function of putative LHNs more directly in polyamine attraction of mated females, we selected several LHN split-Gal4 lines that labeled AD1b2, but also LHNs that were not directly connected to VC5 PNs (Dolan et al., 2019). Using these LHN split-Gal4 lines, we expressed a temperature-sensitive dominant-negative mutant of dynamin, Shibire ${ }^{\text {ts } 1}$ (Kitamoto, 2002) to temporally

150 block synaptic output at $30^{\circ} \mathrm{C}$. All lines and Gal4 or Shibire ${ }^{\text {tsl }}$ only controls were tested in groups in the Tmaze assay at 5-7 days of age (Fig. 1A,G). While we observed a decrease in putrescine preference in several lines, only two lines showed a significant reduction (Fig. 1G). One of these lines, L1779, strongly labeled several neurons of the AD1b2 type suggesting that the observed synaptic connections are indeed relevant for the attraction of putrescine (Fig. S1K). Two additional lines that also expressed in AD1b2 neurons, by

155 contrast, did not reduce putrescine attraction significantly (Fig. S1I,J). These lines, however, labeled fewer neurons than L1779 suggesting a certain redundancy between the AD1b2 type neurons (L1538/1539: 9.8 \pm 1.5 vs. L1779: 19.3 $\pm 2.6, \mathrm{p}<0.001)$. More surprisingly, expression of Shibire ${ }^{\text {ts } 1}$ under the control of line L1614, which labels the aSP-g neuron, also significantly reduced the attraction to putrescine (Fig. 1G). The aSP-g neuron receives input from the DA1 glomerulus (Fig. 1H), which in turn receives input by the male pheromone cVA (11-cis-Vaccenyl Acetate) detecting OSNs.

Taken together, the data indicate that multiple LHONs are involved in the mated female's attraction to polyamines, including AD1b2, which receive strong synaptic input by one type of VC5 PN, and aSP-g neurons, which are activated by cVA as well as some other odors (Kohl et al., 2013).

\section{Mushroom body Kenyon cells mediate state-dependent polyamine attraction}

We next investigated the role of the MB in mating state-dependent polyamine preference due to its role metabolic state-dependent behavioral adaptation and long-term plasticity. We used another set of split-Gal4 lines, generated to target individual cell types of the MB network (Aso et al., 2014a).

We started with the principal cells of the MB, the Kenyon cells (KCs), which long axons form the MB lobes

170 that are innervated by DANs and provide output to MB output neurons (MBONs). To this end, we initially behaviorally analyzed 10 different MB-Gal4 lines expressing in the 7 different subsets of KCs by overexpressing Shibire ${ }^{\text {ts } 1}$. In mated females, blocking KC output at the time of testing resulted in significantly reduced preference for polyamine odor in mated females significantly in 2 of the tested lines suggesting that MB output plays an important role in polyamine preference of mated females (Table 1). To

175 exclude that the higher preference of the mated females resulted from a group effect or social interaction and was not a decision of individual females (Duménil et al., 2016), we also tested single females. We expressed Shibire $^{\text {tsl }}$ in all KCs $\left(M B 10 B-G a l 4 ; U A S-s h i^{\text {tsl }}\right)$, shifted the females to $30^{\circ} \mathrm{C}$, and tested their individual polyamine odor preference in the T-maze assay. Blocking $\mathrm{KC}$ output during the choice test significantly impaired the single female's choice, and mated females became almost indifferent to polyamine (Fig. 2A,B, $50 \%$ represents chance) thus confirming the T-maze group data. By contrast, $\mathrm{KC}$ output did not affect the 
185 Given the role of MB in learning and lasting behavioral adaptations, we wondered whether KC output might be required not only for expression of polyamine odor attraction but also for the increase from virgin to mated female behavior. To test this hypothesis, we shifted the females (MB10B-Gal4;UAS-shits $)$ from 25 to $30^{\circ} \mathrm{C}$ for $24 \mathrm{~h}$ at three different time points: (1) $24 \mathrm{~h}$ before mating, (2) $24 \mathrm{~h}$ during mating, and (3) $24 \mathrm{~h}$ after mating (Fig. 2A). Blocking synaptic output during $24 \mathrm{~h}$ before or between mating and test had no

choice of virgins (Fig. 2B). Similarly, blocking the synaptic output of only $\alpha \beta$-type KCs (MB008BGal4;UAS-shits $)$ or just the surface subset of this KC type $\left(M B 185 B-G a l 4 ; U A S-s h i^{t s I}\right)$ significantly reduced the mated female's preference for polyamine without affecting virgin behavior (Table 1 and Fig. 2C,D). significant effect on the mated female's preference for polyamines (Fig. 2B). However, blocking KC output exclusively during the $24 \mathrm{~h}$ at and around mating, completely prevented the change from virgin to mated female putrescine attraction (Fig. 2B). Activation of $\mathrm{KC}$ output instead of mating using the temperaturesensitive channel TrpA1(Rosenzweig et al., 2008), on the other hand, was insufficient to induce higher polyamine odor attraction in virgin females (Fig. S2A-D). Importantly, all females were analyzed for successful fertilization upon mating and females that did not produce offspring were discarded from the analysis. Thus, KC synaptic output appears to be required at two timepoints: First, it is necessary to induce the change in behavior upon mating, and second, it influences the actual behavioral choice.

Behavioral modulation via the $\mathrm{MB}$ and $\mathrm{KCs}$ relies on neuromodulation and synaptic changes. The activation of type I Ca2+/CaM-dependent adenylyl cyclase (AC) Rutabaga through G-protein coupled receptor signaling, is an evolutionary conserved key mechanism involved in synaptic plasticity, for instance through co-incidence detection of two stimuli or events (Gervasi et al., 2010). In the Drosophila, rutabaga mutant animals fail to form short-term memories of electric shock or sugar reward (Livingstone et al., 1984). We tested whether the increase in putrescine attraction would be triggered in rutabaga mutant females upon mating. To this end, we allowed two days old rutabaga mutant virgin females and controls to mate with wildtype males for $24 \mathrm{~h}$. Five days after mating, 94 percent of control heterozygous females chose polyamine odor over control, while only 62.5 percent of rutabaga females preferred polyamines (Fig. S2E). Virgin females of the same age with or without a functional rutabaga gene chose polyamine odor only 56 percent of the time (Fig. S2E). Thus, mechanisms involved in synaptic plasticity appear to be involved in

210 the preference change of females upon mating.

Together, these data suggest that MB KCs and short-term plasticity mechanisms are required for the matinginduced switch in female choice behavior and decision-making.

215 Behavioral screening pinpoints roles for distinct MB neurons in reproductive state-dependent female decision-making

MBONs, as major output of KCs, are involved in guiding valence-based action selection. Exogenous activation through optogenetics of selected MBONs elicits attraction or aversion, respectively, to light (Aso et al., 2014b). MBON dendrites tile the vertical and horizontal lobes of the MB with their dendrites and

220 thereby provide compartment-specific output of 15 different MB regions (Aso et al., 2014a). In addition, some MBONs receive input by all KCs, while others are specific to KC subsets. We behaviorally screened, using Shibire ${ }^{\text {ts } 1}, 22$ MB-Gal4 lines for MBON requirement at the time of olfactory choice in mated females. From the tested 22 lines, 4 lines significantly impacted female choice (Table 2). Given that LH output is contributing to polyamine odor attraction (see Figure 1), we focused on MBON- $\alpha 2$ sc (i.e. MB080C-Gal4; aka MBON18), which projects from the MB to the LH (Dolan et al., 2018). Inactivation of MBON- $\alpha 2 \mathrm{sc}$ 
during preference testing of mated females significantly reduced their postmating attraction to polyamine (Fig. 3A,B). In comparison, inhibition of $\mathrm{MBON}-\alpha 2 \mathrm{sc}$ output did not change polyamine preference behavior of virgins (Fig. 3C). When MBON- $\alpha 2$ sc output was blocked during mating, we did not observe a significant decrease in polyamine preference (Fig. 3D) indicating that its output is primarily required during behavioral expression.

We next tested whether its activation would modulate the behavior. To this end, we again expressed the temperature sensitive channel dTrpA1 in MBON- $\alpha 2 \mathrm{sc}$ (MB080C-Gal4;UAS-dTrpA1) and activated the neurons by shifting the animals to 30 degrees (Fig. 3E) (Rosenzweig et al., 2008). The neurons were activated in virgin females instead of mating for a period of $24 \mathrm{~h}$. As with the regular mating protocol, we then tested these females for their preference for polyamine odor 4 days after MBON activation. Activation of MBON- $\alpha 2 \mathrm{sc}$ instead of mating induced a mated female-like attraction level of polyamine odor in virgin females (Fig. 3E) indicating that the output of this MBON promotes higher polyamine preference upon mating. Consistent with this observation, EM connectomics data (neuPrint) shows that MBON- $\alpha 2 \mathrm{sc}$ is synaptically directly and indirectly connected to the LHONs AD1b2 and aSP-g, which are equally necessary for polyamine attraction (Fig. 3F,G).

Together, these data indicate that MBON- $\alpha 2 \mathrm{sc}$ activity and possibly its direct and indirect synaptic connections to LHONs AD1b2 and aSP-g are necessary for mated female polyamine odor attraction.

245 The $\beta^{\prime}$ '1and $\beta^{\prime} 2$ compartments of the mushroom body regulate polyamine choice behavior Our screening data further suggested that, in addition to $\alpha \beta$ s-type $\mathrm{KCs}$, synaptic output of $\alpha^{\prime} \beta^{\prime}$ ap-KCs $\left(M B 463 B-G a l 4 ; U A S-s h i^{t s I}\right)$ was required at the time of odor test for mated female preference for polyamines (Table 1). Among the MBONs that receive strong input from $\alpha^{\prime} \beta$ 'ap-KCs is the atypical (i.e. it also receives

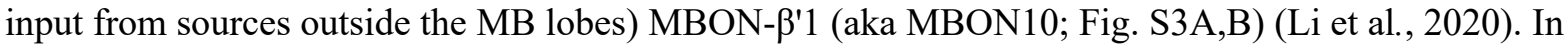

250 contrast to our MBON- $\alpha 2 \mathrm{sc}$ data, inactivation of MBON- $\beta^{\prime} 1$ (MB057B-Gal4;UAS-shits $)$ output at the time of olfactory test did not significantly reduce the mated female's preference for polyamine and also had no effect on virgin female behavior (Table 2 and S3C). Blocking of synaptic output of MBON- $\beta$ ' 1 during the $24 \mathrm{~h}$ period around mating on the other hand, resulted in a strong and significant reduction of polyamine odor preference in these mated females at the time of odor test (Fig. 4A,B) suggesting that this MBON's

255 output critically contributes to the induction of mating-induced behavioral changes but not to the expression. In line with this hypothesis, dTrpA1-mediated activation of MBON- $\beta^{\prime} 1$ in virgins in place of mating significantly increased these females' polyamine preference at the time of choice (Fig. 4C). Equally, activation of MBON- $\beta^{\prime} 1$ in virgin females only at the time of choice also led to a significant increase in polyamine attraction compared to controls (Fig. 4D). This activation also reduced mated female attraction 260 (Fig. 4D), which suggests a complex role of this MBON. Nonetheless, these results argue that MBON- $\beta^{\prime} 1$ synaptic output is required for triggering enhanced polyamine attraction upon mating.

Relatively little is known regarding the function of MBON- $\beta^{\prime} 1$ that would explain the observed behavioral phenotypes. MBON- $\beta^{\prime} 1$ releases GABA as neurotransmitter and might, therefore, inhibit downstream

265 neuronal activity (Aso et al., 2014a). We again used the trans-TANGO system to identify the putatively inhibited downstream neuron (Fig. 4E,E'). Expression of trans-TANGO under the control of MB057B-Ga14 primarily and repeatedly labeled a neuron with neurites in the $\beta^{\prime} 2$ region of the MB (Fig. 4E). We confirmed this observation and identified a candidate neuron for our TANGO result using the EM connectome (Fig. S4A-C) (Li et al., 2020). PAM- $\beta$ '2m (aka PAM06) receives direct input from MBON- $\beta$ ' 1 and anatomically 270 matches the light microscopy images (Fig. S4A). PAM- $\beta$ ' $2 \mathrm{~m}$ in turn synapses onto MBONs providing 
output from the $\beta^{\prime} 2 \mathrm{MB}$ compartment (i.e. $\beta^{\prime} 2 \mathrm{mp}, \beta^{\prime} 2 \mathrm{mp} \_$bilateral; Fig. S4C). Interestingly, $\beta^{\prime} 2 \mathrm{MBONs}$ (i.e. MBON- $\gamma 5 \beta^{\prime} 2$ a) form direct input synapses on MBON- $\beta^{\prime} 1$ (Fig. S4B) suggesting a recurrent loop between the $\beta$ '1, $\beta$ '2 MBONs and DANs (Li et al., 2020). Such a recurrent loop could drive persistent behavioral output as previously shown for male courtship memory (Zhao et al., 2018).

MBONs providing output from $\beta^{\prime} 2$ were shown to be required for hunger state-dependent odor aversion (Lewis et al., 2015; Owald et al., 2015). Moreover, activation of these MBONs using optogenetics is sufficient to elicit strong avoidance (Aso et al., 2014b). Based on these prior data, we postulated that MBON- $\beta^{\prime} 1$ and MBON- $\beta$ ' 2 could modulate each other's activity. To probe the involvement of $\beta$ ' 2 MBONs in polyamine attraction, we chose line MB011B-Gal4, which drives Gal4 expression in three different MBON-types, namely MBON- $\beta^{\prime} 2 \mathrm{mp}, \mathrm{MBON}-\beta^{\prime} 2 \mathrm{mp} \_$bilateral and MBON $\gamma 5 \beta^{\prime} 2 \mathrm{a}$. Indeed, temporary thermogenetic activation of these MBONs (MB011B-Gal4;UAS-dTrpA1) at the time of olfactory choice, significantly reduced the mated female's attraction to polyamine (Fig. 5A,B). Conversely, temporary synaptic output inhibition at the time of choice in virgins increased their attraction to polyamine odor (Fig. 5C). By contrast, neither activation nor synaptic output blockage at the time or in place of mating had an effect of the female's preference behavior (Fig. 5D,E). These data suggest that $\beta^{\prime} 2$ output is crucial for the expression of the choice, but in contrast to $\beta^{\prime} 1$ output, it is insufficient to induce the mating-induced change in choice behavior.

290 Altogether, we propose the involvement of at least three MB compartments, namely, $\beta^{\prime} 1, \beta^{\prime} 2$ and $\alpha 2$ in mating state-dependent odor attraction. These compartments interact through multiple synaptic connections: PAM- $\beta^{\prime} 2$ m connects MBONs of the $\beta^{\prime} 1$ and $\beta^{\prime} 2$ compartments (see Fig. S4C), while EM connectomics suggest that PPL1- $\gamma 2 \alpha^{\prime} 1$ forms a bridge between MBON- $\beta$ ' 1 and MBON- $\alpha 2$ sc (Fig. 5F). Finally, MBON$\alpha 2$ sc synaptically connects these MB circuits to the LH to two types of output neurons driving attraction of

295 mated females to polyamines.

\section{Specific dopaminergic neurons can replace mating experience}

Our data indicated that specific MB compartments and possibly DANs are involved in mating statedependent female odor preference. Moreover, our imaging data provided evidence that the mating-related pheromone cVA activates DANs in a mating state-dependent manner, in particular the DANs innervating the $\alpha^{\prime} 1$ and $\beta^{\prime} 1$ MB compartments (Siju et al., 2020) (Fig. S5A,B). Therefore, we sought to test experimentally whether DANs are involved in the mating-induced change in female polyamine preference. We started with the DANs innervating the $\alpha$-lobe region. To this end, we activated PPL1- $\alpha 2$-type DANs by expressing dTrpA1 under the control of MB058B (only PPL1- $\alpha^{\prime} 2 \alpha 2$ ), MB060B (PPL1- $\alpha^{\prime} 2 \alpha 2$, PPL1- $\alpha^{\prime} 3$, PPL1- $\alpha 3$, PPL1- $\gamma 2 \alpha^{\prime} 1$ ), and MB438B (PPL1- $\alpha^{\prime} 2 \alpha 2$, PPL1- $\alpha^{\prime} 3$, PPL1- $\gamma 1$ pedc) (Fig. S5C). Activation of neurons in neither of these lines instead of mating changed virgin female preference behavior at the time of preference test (Fig. S5D,E). By contrast, activation at mating of neurons labeled by line MB060B, the only among the three that includes PPL1- $\gamma 2 \alpha^{\prime} 1$, reduced mated female attraction at choice test (Fig. S5E).

Therefore, it is conceivable that the MBON-connecting DAN PPL1- $\gamma 2 \alpha^{\prime} 1$ contributes to the mating induced switch in female odor preference. We found, moreover, that blocking the output of these PPL1 DANs at the time of olfactory test, using the same transgenic lines to express Shibire ${ }^{\text {tsl }}$, led to a significant increase in attraction to polyamine odor in virgin females (Fig. 6A-D).

We next analyzed the putative role of PAM DANs innervating the $\beta^{\prime} 1$ and $\beta$ '2 compartments of the MB. We started with line MB188B, because it labeled several PAM DANs with axons in the $\beta$ ' 1 lobe, namely PAM- $\beta$ ' 1 ap and PAM- $\beta$ ' $1 \mathrm{~m}$, as well as PAM- $\gamma 3$, and PAM- $\gamma 4$ (Aso et al., 2014a). We manipulated the 
activity of these PAMs in virgin and mated females as a replacement for actual mating or during mating (Fig. 6E, S6F). Neuronal activation with TrpA1 was highly efficient in switching virgin female preference to mated female preference for polyamine (Fig. 6E) indicating that $\beta$ ' 1 DANs can replace mating experience and possibly respond to mating-related signals; the male pheromone cVA could be such a signal as it induces a calcium increase in $\beta$ '1 DANs (Fig. S5A,B). By contrast, inhibition of synaptic output of the same DANs had not effect (Fig. S6F) indicating that these DANs are sufficient but not strictly necessary for the mating switch to happen. Similar to activation of MBON- $\beta$ '1 (see Fig. 4D), activation of these PAM neurons during mating or at test reduced the effect of mating and mated females showed a significantly lower attraction at the time of testing (Fig. 6E,F). Therefore, it appears that $\beta^{`} 1$ DANs can bidirectionally modulate female choice behavior possibly through alternative pathways (Fig. 6E). For instance, PAM- $\beta{ }^{`} 1$ neurons project to MBON- $\beta^{`} 1$ (MBON10) and MBON- $\gamma 3 \beta^{`} 1$ (MBON09), but also to a PAM- $\beta$ ‘2 (Fig. 6G). To address this, we inhibited synaptic output of all PAM DANs simultaneously by expressing Shibire ${ }^{\text {ts } 1}$ (MB42B, PAM- $\alpha 1$, PAM- $\beta^{\prime} 1$ ap, PAM- $\beta^{\prime} 1 \mathrm{~m}$, PAM- $\beta^{\prime} 2 \mathrm{a}$, PAM- $\beta^{\prime} 2 \mathrm{~m}$, PAM- $\beta$ '2p, PAM- $\beta 1$, PAM- $\gamma 3$, PAM$\gamma 4$, PAM- $\gamma 4<\gamma 1 \gamma 2$, PAM- $\gamma 5$ ), of a large subset (MB316B) or of a different subset of horizontal lobeinnervating DANs (MB109B, PAM- $\beta$ '2a, PAM- $\gamma 5$ ) during olfactory choice test (Fig. S6A). While inhibition of only PAM- $\beta$ '2a, PAM- $\gamma 5$ DANs had no effect, inhibition of all PAM DANs or a large subset (PAM- $\beta^{\prime} 2 a$, PAM- $\beta$ '2m, PAM- $\beta$ '2p, PAM- $\gamma 4$, PAM- $\gamma 4<\gamma 1 \gamma 2$ ) strongly reduced mated female attraction to polyamine odor (Fig. S6B-D). Interestingly, this result was exactly opposite of what we had observed by inhibiting synaptic output of PPL1 DANs at test (see Fig. 6A-D). Moreover, activation of PAM- $\beta$ '2a, PAM- $\gamma 5$ in place of mating, in contrast to activation of PAM- $\beta$ ' 1 DANs, was insufficient to induce an increased attraction of these virgins at the time of test (Fig. S6E).

The present data, therefore, provides support for an important role of DANs innervating the $\beta$ ' 1 region of the MB in inducing an increased attraction of polyamine odors. Given that $\beta$ '1-DANs receive synaptic input from both LHON AD1b2 (Fig. S6G) and aSP-g (Fig. S7A,B), it is possible that these neurons relay matingrelated signals (e.g. cVA, food odor) to the $\mathrm{MB}$ network, thereby inducing a lasting increased attraction of mated females through a, likely, complex pathway of PPL1 and PAM DANs and the identified MBONs.

\section{Putative internal signals for mating state}

345 Male flies perform a complex courtship before attempting to mount the female in order to mate. During this time, females will, among other signals, also smell male-emitted pheromones such as cVA. Interestingly, $\mathrm{cVA}$ and 7-tricosene are transferred onto the female during courtship and mating, and remain on her body for an extended period of time $(\sim 24 \mathrm{~h})$. To test the role of courtship and its signals $v s$. actual mating, we allowed fly couples to go through the courtship ritual but separated them just as the male attempted to mount the female. We then analyzed the odor preference behavior of these females that were technically still virgins. The behavior of these females lay between virgins and mated females and was neither significantly different from virgins nor from mated females consistent with the hypothesis that courtship related signals might contribute as behavioral change triggers (Fig. 7A). Based on this observation and our data above, we next tested one specific aspect of the courtship ritual, odor detection. We reasoned that pheromones such as cVA might influence or trigger the behavioral change, consistent with our data indicating that the aSP-g LHON is required for enhanced putrescine preference (see Fig. 1). Pheromone detection relies on the general OR co-receptor, ORCO, and different specific ORs. We thus analyzed mated females mutant for $O R C O$, and hence unable to smell pheromones among other odors. Importantly, ORCO is not required for the detection of polyamine odor, which is mediated by IR76b and IR41a (Hussain et al., 2016b; Silbering et al., 2011). Mated $O R C O$ mutant females displayed a significantly lower polyamine preference compared to 
controls (Fig. 7B). Previous work showed that food odors enhance courtship and mating (Grosjean et al., 2011), and that cVA promotes aggregation behavior of flies on food patches (Das et al., 2017; Lebreton et al., 2015). Interestingly, polyamines are present in high concentrations in standard fly food: histamine (5.49), ethanolamin (676.65), phenylethylamin (5.16), putrescine (172.50), $\beta$-alanine (930.84), tyramine (166.64), spermidine (18120.77) and spermine (492.81) (Fig. S8A). Thus, the total amount of polyamines in standard fly food is $\sim 20 \mathrm{mg}$ in $100 \mathrm{~g}$ food, which is comparable to levels in very polyamine-rich foods such as oranges (Kalac, 2014).

To test whether the presence of odors such as pheromones or polyamines was important during mating to induce a higher polyamine odor preference upon mating, we blocked the synaptic output of ORCO and IR76b/IR41a-expressing OSNs exclusively during the $24 \mathrm{~h}$ at and around mating (Fig. 7C). While synaptic inhibition of IR41a- and IR76b-expressing OSNs during mating had no effect on the mated female's odor preference, blocking the synaptic output of ORCO-OSNs during mating but not at test significantly reduced those females' attraction to the putrescine odor (Fig. 7C). Next, to test whether the context of food was necessary for females to change their preference upon mating, we removed virgin females for $24 \mathrm{~h}$ from fly

375 food and transferred them to a pure agarose substrate. We mated $50 \%$ of these females to males on agarose, while the other $50 \%$ remained alone - hence, they remained virgin - for the same amount of time. After these $24 \mathrm{~h}$, flies were transferred again onto regular fly food. Virgins behaved as expected and showed no polyamine preference (Fig. S8B). Females mated on agarose showed no significant decrease in polyamine preference compared to females mated on regular fly food (Fig. S8B). We next raised and mated females on a holidic diet containing precise amounts of nutrients such as amino acids but no added polyamines (Piper et al., 2014). We again found no difference in the preference for polyamine odor between the two groups of mated females (Fig. S8C).

These results provide evidence that courtship promoting signals such as pheromones contribute to a switch in preference behavior, but that the presence of food odor, including polyamines, during mating is not

385 required.

We continued to look for additional triggers that could work in synergy with courtship such as signals transferred or present during mating. During copulation the male fly transfers sperm and seminal fluid into the female reproductive tract. Among the factors transferred with the sperm is one of the ligands of SPR, sex

390 peptide (SP). SP binds to neurons expressing SPR in the female's reproductive tract and induces the socalled canonical post-mating switch, a suite of behaviors associated with reproduction such as increase in egglaying and rejection of males attempting to mate (Kubli, 2003; Yapici et al., 2008). Although SPR is required for the change in the mated female's polyamine preference behavior, this appears to rely mainly on the other ligands, myoinhibitory peptides (MIPs) (Hussain et al., 2016a; Kim et al., 2010) as females mated 395 to SP mutant males still undergo the change in polyamine preference behavior (Hussain et al., 2016a). Therefore, we next tested females mated to sterile males. There are two previously used experimental methods to achieve such an effect: males that do not produce either seminal fluid protein or sperm (Boswell and Mahowald, 1985; Chow et al., 2015). To generate males incapable of producing seminal fluid protein, accessory glands were disrupted through induction of ER stress (see methods; prd-GAL4;UAS-BiP-RNAi). After copulation with a seminal fluid protein deficient male, females showed a normal increase in preference for polyamine odor as compared to controls (Fig. 7D). Similarly, copulation with sperm-deficient males, which were generated by using mutants of tudor (Chapman et al., 2003), also led to mated females that behaved indistinguishable from females that mated with wildtype males (Fig. 7E). This result was in line with our previous finding (Hussain et al., 2016a), and suggested that not only was SP not essential, but also that other sperm- and seminal fluid-associated factors were dispensable. To further support the 
conclusion that fertilization was not essential for the change in odor preference, we tested females that, similarly to females mated to sterile males, were unable to produce eggs by generating ovoD mutant females. $O v o D$ females mated with wildtype males underwent the switch in behavior and were attracted to polyamines to the same extent as controls (Fig. 7F).

Altogether these data point to a role for multiple signals including courtship signals, and possibly the physical act of mating itself in inducing the mated female's increased preference for polyamines.

\section{DISCUSSION}

The transition from a sexually immature to a mature, reproducing parent is a major step in the life of most animals. With this step come new needs and demands, reflected in a significant change in an animal's behavior and preferences. While many aspects of reproductive behavior are innate, sexual and parental behavior is highly flexible and changes through physiology and with experience (Griffith and Ejima, 2009; Koch and Ehret, 1989; Marlin et al., 2015). In addition, sexually mature animals spend a major proportion of their lives with generating or caring for their offspring. In line with this, some of the behavioral and neuronal changes persist for a significant fraction of an animal's lifespan (Hoekzema et al., 2017; Insel et al., 1995; Reisenman et al., 2009; Zhao et al., 2017).

We have previously shown that mating induces a MIP/SPR-dependent neuromodulation of olfactory neuron output, which is sufficient to induce the mated female's heightened attraction to an important nutrient (Hussain et al., 2016a; Hussain et al., 2016b). Here, we have identified important neural circuit elements required for this attraction. The involvement of the LH and MB, two important olfactory brain centers involved in stereotyped and experience-dependent odor responses, respectively, suggest that a distributed, recurrently connected neural network underpins the mating state-dependent adaptation in female preference behavior (Fig. 8).

\section{Distributed processing underpins state-dependent behavioral change}

Soon after mating, a female fly starts to look for places to lay her eggs. Although this behavior is generally viewed as innate, it is induced by mating and, possibly, the experiences surrounding it. Interestingly, earlier work has shown that oviposition decisions can require the output of the MB (Azanchi et al., 2013).

435 Furthermore, MB output is necessary for metabolic state-dependent innate odor behavior (Bracker et al., 2013; Lewis et al., 2015; Sayin et al., 2019; Tsao et al., 2018). We now show that MB output is also required for reproductive state-dependent behavior; first, at the time of mating, and later when the female makes the actual decision to move toward high polyamine.

440 Our data further suggests that the activity of DANs of the PPL1 and PAM clusters contributes significantly to the decision of the female. For instance, inhibition of PPL1 DANs in virgin females at the time of olfactory choice ( $\sim 5$ days after mating) strongly increased those virgins' attraction to polyamines. Vice versa, activation of PPL1 DANs during mating significantly reduced the mated female's interest in polyamine odor when tested several days later (see Fig. 6). Conversely, the activation of PAM cluster DANs innervating the $\beta$ ' 1 region of the MB was sufficient to induce mated female polyamine choice behavior in virgin females, while inhibition of MBON- $\beta$ ' 1 output at the time of mating prevented the switch to mated female behavior. Similarly, activation of MBON- $\beta$ ' 1 output instead of mating also increased the virgin's polyamine attraction to mated female levels. We propose that mating-induced modulation of the relative activity of DANs and connected MB lobes (in particular of the $\beta$ ' 1 region) changes the female's interest in the beneficial compound polyamine. Notably, $\beta$ ' 1 MBONs have been implicated previously in courtship 
conditioning in Drosophila males (Montague and Baker, 2016). At this point, we can only speculate about what $\beta^{\prime} 1 \mathrm{MB}$ output does to these reproduction related behaviors. EM connectomics and trans-TANGO data suggest that $\beta$ '2 DANs are directly and $\beta$ '2 MBONs indirectly connected to MBON- $\beta$ ' 1 . In line with this, we find that blocking $\beta$ '2 MBON output indeed increases the virgin's attraction to polyamine.

The other MB area of functional importance is the $\alpha 2$-lobe region. Based on our results we propose MBON$\alpha 2 s c$ allows MB output to influence LH output, similar to the mechanism described by Dolan et al. previously (Dolan et al., 2018). MBON- $\alpha 2$ sc, also known as MB-V2 $\alpha$, is involved in memory recall of taste memory (Kirkhart and Scott, 2015) and olfactory memory (Sejourne et al., 2011). Hence, in the present scenario, MB output might regulate the expression of polyamine-induced LH-dependent behavior according to mating state. Interestingly, connectomic analysis shows that MBON- $\beta$ ' 1 provides indirect input -via PPL1- $\gamma 2 \alpha^{\prime} 1$ and another neuron- to MBON- $\alpha 2 \mathrm{sc}$. It is, therefore, conceivable that MBON- $\beta$ ' 1 modulates the output of MBON- $\alpha 2 \mathrm{sc}$ and in turn polyamine attraction.

In the context of classical aversive odor conditioning, two LH output neuron (LHON) cell types, PD2a1 and b1, are postsynaptic to MBON- $\alpha 2 s c$ (Dolan et al., 2018). These LHONs are required for innate attraction to food odorant. While the same LHONs are redundant in the present paradigm (see Fig. 1G, line L989), using imaging, connectomics and behavioral screening, we find that polyamine attraction involves at least two types of LHONs, namely AD1b2 and aSP-g neurons (see Fig. 1G). According to neuPrint, AD1b2 neurons

470 receive direct synaptic input from MBON- $\alpha 2 \mathrm{sc}$, while aSP-g and MBON- $\alpha 2 \mathrm{sc}$ are bidirectionally connected through another LH neuron, LHCENT.

Taken together, we propose that a network of neurons primarily innervating the $\alpha 2, \beta^{\prime} 1$ and $\beta^{\prime} 2$ regions of the MB lobes and specific neurons in the LH controlling mating state-dependent preference of females to

475 important nutrients for reproduction. The proposed model agrees with earlier findings that avoidance of $\mathrm{CO}_{2}$, which requires the output of specific LHONs (Varela et al., 2019), can be suppressed in a metabolic state-dependent manner by specific KCs and MBONs (Bracker et al., 2013; Lewis et al., 2015).

\section{Triggers for reproductive state-dependent changes in the female brain}

480 We have previously shown that SP appears to play a rather redundant role in the increase in polyamine attraction (Hussain et al., 2016a). Instead, SPR in OSNs together with its conserved ligand MIP depress OSN synaptic output upon mating and thereby trigger the switch to higher polyamine attraction. How this signaling is triggered by mating remained unclear. Surprisingly, courtship without copulation increased virgin female polyamine preference to a level not significantly different from mated females. In line with

485 this result, a temporal blocking of synaptic output of ORCO-dependent OSNs during mating reduced the females' attraction suggesting that OR-dependent odor detection during mating, for instance male pheromone, contributes to the change in behavior from virgin to mated female. Of note, males transfer some of their pheromone onto the female during copulation (Keleman et al., 2012), and they also mark feeding and oviposition sites for females by depositing pheromone (Lin et al., 2015), suggesting that pheromones

490 are present in the female's environment just before, during and sometime after mating (Ejima et al., 2007). By contrast, the presence of polyamines during mating was not required as temporal inhibition of polyamine OSN synaptic output during mating did not affect the mated female's high putrescine attraction during test. In line with a putative role of odors such as pheromones, in vivo calcium imaging data shows that the pheromone cVA activates DANs, and in particular, $\beta$ ' 1 innervating DANs, in a mating state-dependent

495 manner (Siju et al., 2020). Activation of $\beta$ '1 DANs was sufficient to replace mating in inducing mated 
female levels of polyamine odor attraction in virgins. We, hence, speculate, that the mechanisms we describe here might represent a female version of male courtship learning (Ejima et al., 2007; Keleman et al., 2012). Consistent with our observations, few DANs can mimic courtship experience in naive males in the absence of an actual rejection by an already mated female (Keleman et al., 2012). Males likely associate $\mathrm{cVA}$, which was transferred during previous copulation by another male onto the female, with the experience of rejection by an already mated female (Ejima et al., 2007). In our scenario, the pheromones transferred to the female during copulation might modify the female's behavior, too. In addition, mating itself stimulates mechanosensory neurons in the female's reproductive tract and thereby appears to contribute to a switch in her behavior (Gou et al., 2014; Shao et al., 2019). Other hormonal or peptidergic signals, such as steroids or juvenile hormone (JH) could play a role (Moshitzky et al., 1996). For instance, $\mathrm{JH}$ drives network maturation of the MB in young flies and thereby promotes their ability to form memories (Leinwand and Scott, 2021). Recent elegant work showed that mating triggers neuropeptide (i.e. Bursicon) release from enteroendocrine cells, which in turn modulate female appetite (Hadjieconomou et al., 2020). Given the presence and partial involvement of many triggers such as mating, courtship, feeding, it is

510 possible that, as our data indicates, multiple factors trigger the lasting behavioral change observed in mated females.

\section{Does reproduction induce neural network changes to facilitate reproductive behaviors?}

A state change, such as before and after mating, could conceivably represent a window of opportunity for 515 changes in relevant neural networks (Griffith and Ejima, 2009) or even globally in many or all brain regions. Our in vivo whole brain imaging data suggests that, at least in flies, mating does not induce a global change in brain state. Rather, mating appears to induce plasticity in a distributed network spanning multiple brain regions. In line with our data, mating and SPR signaling were recently shown to enhance MB-dependent long-term memory in Drosophila females (Scheunemann et al., 2019). Similar situations as we have

520 characterized here might arise at times of hunger (Krashes et al., 2009; Root et al., 2011). In other words, food consumed when hungry not only tastes and smells better, it also leaves a longer lasting impression. Mechanistically, such a window of enhanced plasticity might arise through an increase in cAMP in neurons of the MB network at critical phases of life (Louis et al., 2018).

Beyond insects, such a scenario could arise during different periods of important changes in the body 525 (Carlson et al., 2018). An example could be mother-child-bonding in mammals where mother and newborn are particularly sensitive to memorizing the sensory characteristics of each other for a certain period after birth (Cernoch and Porter, 1985; Galbally et al., 2011; Sullivan, 2003). For instance, the neuropeptide oxytocin, which is released during birth and lactation, induces lasting changes in the mother's cortex, transforming weaker responses to pup calls in the virgin to strong responses in mothers (Marlin et al., 2015).

530 The orchestration of internal state-dependent sensory tuning with plasticity of neurons in higher brain centers could, thus, ensure that animals remember the most relevant information at key turning points in their lives. 


\section{MATERIALS and METHODS}

\section{KEY RESOURCES TABLE}

\begin{tabular}{|c|c|c|}
\hline REAGENT or RESOURCE & SOURCE & IDENTIFIER \\
\hline \multicolumn{3}{|l|}{ Antibodies } \\
\hline Anti-Mouse Alexa488 & Molecular Probes & AB_221544 \\
\hline Anti-Mouse Alexa633 & Molecular Probes & AB_141431 \\
\hline Anti-Rabbit Alexa568 & Molecular Probes & AB_141416 \\
\hline Anti-Rabbit Alexa633 & Molecular Probes & AB_2535731 \\
\hline Anti-Rat Alexa568 & Molecular Probes & AB_141874 \\
\hline Mouse monoclonal anti-ChAT & (Yasuyama et al., 1995) & $\mathrm{N} / \mathrm{A}$ \\
\hline Mouse monoclonal anti-OA & Jena Bioscience & AB_2315000 \\
\hline Rabbit polyclonal anti-dsRed & Clontech & AB_10013483 \\
\hline Rabbit polyclonal anti-Tyr & Millipore & AB_11215460 \\
\hline Rat monoclonal anti-GFP [3H9] & Chromotek & AB_10773374 \\
\hline Rat monoclonal anti-Ncadherin & DSHB & AB_528121 \\
\hline \multicolumn{3}{|l|}{ Data } \\
\hline \multicolumn{3}{|l|}{$\begin{array}{l}\text { All original data is available upon } \\
\text { request. }\end{array}$} \\
\hline \multicolumn{3}{|c|}{ Experimental Models: Organisms/Strains } \\
\hline D.mel/Canton-S & Bloomington DSC & Flybase: FBst0064349 \\
\hline D.mel/Dop1R1 attP & Gift from Vanessa Ruta & N/A \\
\hline D.mel/Dop1R2 ${ }^{\text {attP }}$ & (Keleman et al., 2012) & FlyBase: FBal0283280 \\
\hline D.mel/GMR58E02-Gal4 & Bloomington DSC & FlyBase: FBst0041347 \\
\hline D.mel/GMR64C08-Gal4 & Bloomington DSC & FlyBase: FBst0039299 \\
\hline D.mel/GMR95A10-LexA & Bloomington DSC & FlyBase: FBst0061633 \\
\hline D.mel/Gr43a-Gal4 & Miyamoto et al., 2012 & Flybase: FBti0168340 \\
\hline D.mel/Gr5a-Gal4 & Bloomington DSC & Flybase: FBst0057592 \\
\hline D.mel/LexAop2-mCD8-GFP & Bloomington DSC & FlyBase: FBst0056182 \\
\hline D.mel/LexAop-P2X2 & Bloomington DSC & FlyBase: FBst0076030 \\
\hline D.mel/MB10B & Janelia RC & Flybase: FBst0068293 \\
\hline D.mel/MB112C & Janelia RC & FlyBase: FBst0068263 \\
\hline D.mel/MB113C & Janelia RC & FlyBase: FBst0068264 \\
\hline D.mel/MB11B & Janelia RC & Flybase: FBst0068294 \\
\hline D.mel/MB131B & Janelia RC & Flybase: FBst0068265 \\
\hline D.mel/MB152B & Janelia RC & Flybase: FBst0068266 \\
\hline D.mel/MB185B & Janelia RC & FlyBase: FBst0068267 \\
\hline D.mel/MB18B & Janelia RC & Flybase: FBst0068296 \\
\hline D.mel/MB210B & Janelia RC & Flybase: FBst0068272 \\
\hline D.mel/MB22B & Janelia RC & FlyBase: FBst0068298 \\
\hline D.mel/MB242A & Janelia RC & Flybase: FBst0068307 \\
\hline D.mel/MB27B & Janelia RC & Flybase: FBst0068301 \\
\hline D.mel/MB28B & Janelia RC & N/A \\
\hline D.mel/MB298B & Janelia RC & Flybase: FBst0068309 \\
\hline
\end{tabular}




\begin{tabular}{|c|c|c|}
\hline D.mel/MB2B & Janelia RC & Flybase: FBst0068305 \\
\hline D.mel/MB310C & Janelia RC & Flybase: FBst0068313 \\
\hline D.mel/MB320C & Janelia RC & FlyBase: FBst0068253 \\
\hline D.mel/MB355B & Janelia RC & $\mathrm{N} / \mathrm{A}$ \\
\hline D.mel/MB364B & Janelia RC & Flybase: FBst0068318 \\
\hline D.mel/MB370B & Janelia RC & Flybase: FBst0068319 \\
\hline D.mel/MB371B & Janelia RC & Flybase: FBst0068383 \\
\hline D.mel/MB399B & Janelia RC & Flybase: FBst0068369 \\
\hline D.mel/MB417B & Janelia RC & Flybase: FBst0068321 \\
\hline D.mel/MB418B & Janelia RC & Flybase: FBst0068322 \\
\hline D.mel/MB419B & Janelia RC & Flybase: FBst0068323 \\
\hline D.mel/MB433B & Janelia RC & Flybase: FBst0068324 \\
\hline D.mel/MB434B & Janelia RC & Flybase: FBst0068325 \\
\hline D.mel/MB438B & Janelia RC & Flybase: FBst0068326 \\
\hline D.mel/MB461B & Janelia RC & Flybase: FBst0068327 \\
\hline D.mel/MB463B & Janelia RC & Flybase: FBst0068370 \\
\hline D.mel/MB477B & Janelia RC & Flybase: FBst0068328 \\
\hline D.mel/MB504B & Janelia RC & Flybase: FBst0068329 \\
\hline D.mel/MB50B & Janelia RC & Flybase: FBst0068365 \\
\hline D.mel/MB51B & Janelia RC & Flybase: FBst0068275 \\
\hline D.mel/MB52B & Janelia RC & $\mathrm{N} / \mathrm{A}$ \\
\hline D.mel/MB542B & Janelia RC & Flybase: FBst0068372 \\
\hline D.mel/MB543B & Janelia RC & Flybase: FBst0068335 \\
\hline D.mel/MB549C & Janelia RC & Flybase: FBst0068373 \\
\hline D.mel/MB552B & Janelia RC & $\mathrm{N} / \mathrm{A}$ \\
\hline D.mel/MB57B & Janelia RC & Flybase: FBst0068277 \\
\hline D.mel/MB58B & Janelia RC & Flybase: FBst0068278 \\
\hline D.mel/MB5B & Janelia RC & Flybase: FBst0068306 \\
\hline D.mel/MB60B & Janelia RC & Flybase: FBst0068279 \\
\hline D.mel/MB74C & Janelia RC & Flybase: FBst0068282 \\
\hline D.mel/MB80C & Janelia RC & FlyBase: FBst0068285 \\
\hline D.mel/MB82C & Janelia RC & Flybase: FBst0068286 \\
\hline D.mel/MB83C & Janelia RC & Flybase: FBst0068287 \\
\hline D.mel/MB8B & Janelia RC & FlyBase: FBst0068291 \\
\hline D.mel/MB93C & Janelia RC & Flybase: FBst0068289 \\
\hline D.mel/MB9B & Janelia RC & Flybase: FBst0068292 \\
\hline D.mel/L166 & Janelia RC & gift of Greg Jefferis \\
\hline D.mel/L290 & Janelia RC & gift of Greg Jefferis \\
\hline D.mel/L542 & Janelia RC & gift of Greg Jefferis \\
\hline D.mel/L989 & Janelia RC & gift of Greg Jefferis \\
\hline D.mel/L1293 & Janelia RC & gift of Greg Jefferis \\
\hline D.mel/L1479 & Janelia RC & gift of Greg Jefferis \\
\hline D.mel/L1614 & Janelia RC & gift of Greg Jefferis \\
\hline D.mel/L1538 & Janelia RC & gift of Greg Jefferis \\
\hline
\end{tabular}




\begin{tabular}{|c|c|c|}
\hline D.mel/L1539 & Janelia RC & gift of Greg Jefferis \\
\hline D.mel/L1779 & Janelia RC & gift of Greg Jefferis \\
\hline D.mel/L2446 & Janelia RC & gift of Greg Jefferis \\
\hline D.mel/L2382 & Janelia RC & gift of Greg Jefferis \\
\hline D.mel/pBDP-Gal4U (,empty-Gal4') & Bloomington DSC & Flybase: FBst0068384 \\
\hline D.mel/Tdc2-Gal4 & Bloomington DSC & FlyBase: FBst0009313 \\
\hline D.mel/TH-C1-Gal4 & (Liu et al., 2012b) & FlyBase: FBtp0083567 \\
\hline D.mel/TH-D1-Gal4 & (Liu et al., 2012b) & FlyBase: FBtp0083568 \\
\hline D.mel/TH-F1-Gal4 & (Liu et al., 2012b) & FlyBase: FBtp0083570 \\
\hline D.mel/TH-Gal4 & Bloomington DSC & FlyBase: FBst0008848 \\
\hline D.mel/UAS-CsChrimson & Bloomington DSC & FlyBase: FBst0055134 \\
\hline D.mel/UAS-DenMark & Bloomington DSC & FlyBase: FBst0033062 \\
\hline D.mel/UAS-GCaMP6f & Bloomington DSC & FlyBase: FBst0042747 \\
\hline D.mel/UAS-mCD8-GFP & Bloomington DSC & FlyBase: FBst0030001 \\
\hline D.mel/UAS-Shibire ${ }^{\text {ts1 }}$ & Bloomington DSC & FlyBase: FBst0044222 \\
\hline D.mel/UAS-syt-GFP & Bloomington DSC & FlyBase: FBst0006926 \\
\hline D.mel/w1118 & Bloomington DSC & Flybase: FBst0003605 \\
\hline \multicolumn{3}{|l|}{ Software and Algorithms } \\
\hline Matplotlib 1.4 .2 & (Hunter, 2007) & matplotlib.org \\
\hline Numpy 1.8 & (Travis, 2006) & numpy.org \\
\hline Prism 6 and 7 & GraphPad & $\begin{array}{l}\text { graphpad.com/scientific- } \\
\text { software/prism }\end{array}$ \\
\hline Python 2.7 & (van Rossum, 1995) & python.org \\
\hline Pyvttbl 0.5.2.2 & $($ Lew, 2011) & github.com/rogerlew/pyvttbl \\
\hline Scipy.stats 0.14 & (Jones et al., 2001) & scipy.org \\
\hline pCLAMP 10.3 & Molecular Devices & moleculardevices.com \\
\hline Igor Pro 6.37 & Wave Metrics & wavemetrics.com \\
\hline NeuroMatic 3.0 & $\begin{array}{l}\text { (Rothman and Silver, } \\
\text { 2018) }\end{array}$ & neuromatic.thinkrandom.com/ \\
\hline LAS AF E6000 and LAS X & Leica Microsystems & leica-microsystems.com \\
\hline FV10-ASW & Olympus & olympus-lifescience.com \\
\hline
\end{tabular}

\section{Fly husbandry}

Flies were kept at $25{ }^{\circ} \mathrm{C}$ at $60 \%$ humidity with a day:night cycle of 12 hours each. Flies were raised on

545 standard cornmeal medium. Mushroom body lines were received from the Janelia Fly-Light Split-GAL4 Driver Collection (Aso et al., 2014a). BiP, prd lines were a generous gift from Anne von Philipsborn, transTANGO lines were a gift from the Barnea Lab, LH lines were kindly provided by Greg Jefferis.

\section{Experiments with wildtype flies}

550 After eclosion, female virgins were kept in a vial until testing one week later. A second group of female flies were kept with males for $24 \mathrm{~h}$. Then, the males were removed and the females were kept separately in a fresh vial until final testing 4-5 days later. For virgins and mated females, vials were checked for larvae to ensure mating did not or did take place, respectively. 


\section{Shibire and $\mathbf{d T p} A 1$ experiments}

For shibire ${ }^{\text {ts1 }}$ - and dTrpA1 -silencing or -activation, respectively, flies were shifted to a temperature of 30$32{ }^{\circ} \mathrm{C}$ for time frames indicated in the results section.

For temperature shifts at mating, 1-2 day old virgin females were put on $30{ }^{\circ} \mathrm{C}$ for 30 minutes before experienced CantonS males were added for $24 \mathrm{~h}$ at $30{ }^{\circ} \mathrm{C}$ to allow mating during this time period. The males were removed after $24 \mathrm{~h}$. Females were tested 3-5 days later for putrescine preference behavior at $25{ }^{\circ} \mathrm{C}$ in a T-maze assay. The vials were checked for offspring to ensure mating had taken place. For virgin experiments, virgins were kept for $24 \mathrm{~h}$ at $30{ }^{\circ} \mathrm{C}$ without males. This was followed 3-5 days later for putrescine preference behavior at $25{ }^{\circ} \mathrm{C}$ in a T-maze assay.

For temperature shifts at testing, 1 day old females were kept with males for $24 \mathrm{~h}$ at $25^{\circ} \mathrm{C}$. Then, the males

565 were removed and females were tested 3-5 days later for putrescine preference behavior. Virgins were not exposed to males. Flies were tested in a preheated T-maze chamber at $30{ }^{\circ} \mathrm{C}$ and $60 \%$ humidity.

\section{T-maze Experiments}

The two-choice population assay or T-maze was performed as previously described in (Lewis et al., 2015).

570 T-maze tubes were either prepared with $50 \mu 1$ of $100 \mathrm{mM}$ 1,4-Diaminobutan (Putrescine) or $50 \mu 1$ ultrapure water on a piece of Whatman filter paper and sealed with Parafilm until right before the experiment started. Briefly, flies were tested in groups of $\sim 30$ in a non-aspirated T-maze and were allowed $1 \mathrm{~min}$ to respond to stimuli. Experimentation was carried out in climate-controlled boxes at $32^{\circ} \mathrm{C}$ and $60 \% \mathrm{rH}$. A preference index (PI) was calculated by subtracting the number of flies on the control side by the number of flies on the

575 stimulus side and normalizing by the total number of flies. The data is represented by bar graphs of the PIs plus standard error of the mean (SEM) and analyzed using a Mann-Whitney-U test in R-software. This has been done in Excel's RealStats Resource Pack. Unless indicated otherwise, significance stars or n.s. concern the comparison between the two virgin groups or the two mated fly groups, respectively.

\section{Courtship Experiments}

Male flies were kept together in a vial for 2 days until exposure to 1-2 days old virgin females. Always one male was put into a vial with one female. The process of courtship as described in previous studies (Dickson, 2008) was monitored until the male began licking the female and making first attempts for copulation. Immediately, the male was removed from the vial. The female was tested 3-5 days later in a Tmaze for putrescine attraction or avoidance behavior at $25{ }^{\circ} \mathrm{C}$.

\section{Immunohistochemistry}

Trans-TANGO flies were kept for one week at $25{ }^{\circ} \mathrm{C}$ followed by another two weeks at $18{ }^{\circ} \mathrm{C}$, as similarly described in the original paper (Talay et al., 2017). Flies were anaesthetized on ice, put in a glass staining 590 cup with $80 \%$ ethanol. After $30 \mathrm{sec}$ flies were put in another glass staining cup filled with 1x phosphate buffered saline (PBS). Flies were dissected under the microscope and brains were stored in a PCR cap with $1: 4$ solution of $4 \%$ paraformaldehyde (PFA) and $0.1 \%$ phosphate buffered triton (PBT) until final fixation. Brains were fixed in PFA and a drop of PBT for $60 \mathrm{~min}$ at room temperature. Brains were then washed with PBT three times for $20 \mathrm{~min}$ at room temperature. The PBT was removed and replaced by $3 \%$ normal goat serum (NGS) for $30 \mathrm{~min}$ at room temperature. The first antibody mix was incubated in 1:200 with $\alpha$-GFP (mouse), $\alpha$-RFP (rabbit), $\alpha$-NCAD (rat )and $3 \%$ NGS for $24 \mathrm{~h}$ at $4{ }^{\circ} \mathrm{C}$ in darkness. Brains were washed with PBT for $5 \mathrm{sec}$ and then three times for $20 \mathrm{~min}$ at $4{ }^{\circ} \mathrm{C}$ in darkness. The second antibody mix was incubated at 1:200 with $\alpha$-mouse alexa488, $\alpha$-rabbit Cy3, $\alpha$-rat alexa633 and $3 \% \mathrm{NGS}$ for $24 \mathrm{~h}$ at $4{ }^{\circ} \mathrm{C}$ in darkness. 
Brains were washed with PBT for $5 \mathrm{sec}$ and then three times for 20 min at $4{ }^{\circ} \mathrm{C}$ in darkness. After washing with PBT for one last time for $1 \mathrm{~h}$ at $4{ }^{\circ} \mathrm{C}$ in darkness, brains were mounted on a glass slide with VectaShield. Imaging was carried out at a Leica SP8 confocal microscope. Image Processing and analysis have been performed using Fiji software (Schindelin et al., 2012).

\section{In vivo 2-Photon calcium imaging}

605 All imaging experiments were conducted with a two-photon microscope. 5- to 7-day-old female virgin or mated flies of the genotype MB188B-Gal4;UAS-GCaMP6f for PAM neuron imaging and MB057B-Gal4; UAS-GCaMP6f for MBON imaging were used. In vivo fly preparations were prepared according to a method described previously. Preparations were imaged using an Olympus FV1000 two-photon microscope system with a BX61WI microscope and a 40 x , 0.8 water immersion objective. GCaMP fluorescence was

610 excited at $910 \mathrm{~nm}$ by a mode-locked Ti:Sapphire Mai Tai DeepSee laser. Time series images were acquired at $256 \times 256$ pixel resolution at a rate of 2 frames per second for 40 frames using the Olympus FV10-ASW imaging software. In order to minimize the brain movement of in vivo preparations under the two-photon microscope, a drop of $1 \%$ low melting temperature agarose (NuSieveGTG, Lonza) in imaging buffer maintained at $37^{\circ} \mathrm{C}$ was added to the exposed brain. A custom-made odor delivery system with mass flow

615 controllers was used for vinegar stimulation. The odor was delivered in a continuous airstream (1000 $\mathrm{mL} / \mathrm{min}$ ) through a $8-\mathrm{mm}$ Teflon tube placed $\sim 1 \mathrm{~cm}$ away from the fly. In vivo two-photon time series data were acquired at a rate of $\sim 4$ frames/s with 175 x 175 pixel resolution. Changes in fluorescence intensity were measured in manually drawn regions of interest (ROI) using the LAS AF E6000 Lite, LAS X or the Olympus FV10-ASW software. Relative changes in fluorescence intensity were defined as $\Delta \mathrm{F} / \mathrm{F}=100^{*}\left(\mathrm{~F}_{\mathrm{i}}\right.$

$\left.620-\mathrm{F}_{0}\right) / \mathrm{F}_{0}$ for the $\mathrm{i}$ frames after stimulation. Fluorescence background, $\mathrm{F}_{0}$, is the average fluorescence of 60 frames (1 min; ex vivo), 5 frames ( 1 s; in vivo, two-photon), 20 frames ( 1 s; in vivo, ATP static) or 17 frames ( $\sim 1 \mathrm{~s}$; in vivo, ATP perfused). Pseudocolored images were generated using a custom-written MATLAB program and ImageJ.

\section{In vivo light field calcium imaging}

Female flies (nsyb-Gal4;UAS-Gcamp6s) were collected at eclosion to obtain virgins or mated for $48 \mathrm{~h}$ with males of the same genotype. After the males were removed from the mated group, the labels of both groups were blinded to avoid bias. Successful mating or virginity was confirmed later by keeping the vials at $25^{\circ} \mathrm{C}$ and checking for larvae a few days after the experiment. Flies were prepared for whole brain imaging at Day

6304 after eclosion, as previously described (Woller et al., 2021). The light field microscope was set up according to (Aimon et al., 2019). The system was based on a Thorlabs Cerna with a Leica HC FLUOTAR L 25x/0.95 objective and MLA-S125-f12 microlens array (RPC photonics). The microlens array was placed on the image plane, while the camera imaged the microlens array through 50mmf/1.4 NIKKOR-S Nikon relay lenses. The light field images were recorded at $10 \mathrm{~Hz}$ with a scientific CMOS camera (Hamamatsu

635 ORCA-Flash 4.0). For odor delivery, a Syntech Stimulus Controller (CSS-55) was connected to a $4 \mathrm{~mm}$ PVC tube that was placed under the microscope, $5 \mathrm{~mm}$ in front of the fly's head and delivered a constant air stream of $1000 \mathrm{ml} / \mathrm{min}$. Flies were stimulated by redirecting 30\% of main air flow for $1 \mathrm{sec}$ through a headspace glass vial filled with $1 \mathrm{ml}$ of $100 \mathrm{mM}$ putrescine by a manual trigger. Each fly was stimulated five times with $50 \mathrm{sec}$ interstimulus intervals. The imaging data was processed as described in (Siju et al., 2020). 640 Volumes were reconstructed using a python program developed by Broxton et al. (2013) (Broxton et al., 2013) and available on github: https://github.com/sophie63/FlyLFM. The movement artifacts were removed by $3 \mathrm{D}$ registration using the $3 \mathrm{~d}$ volreg routine from AFNI. The following steps were performed with MATLAB (version R2019b, MathWorks Inc.) unless stated otherwise. The voxel time series were 
transformed to DF/F by subtracting and normalizing by a moving average over $20 \mathrm{~s}$ and noise was reduced, using Kalman filtering. Functional regions were then extracted using principal component analysis and independent component analysis. These were then used as landmarks to align the recordings to anatomical templates from (Ito et al., 2014) in FIJI. Masks were created to extract and average DF/F time series for twelve major brain regions (neuropil supercategories). Linear regression of the response time series with the stimulus time series (convolved with the response profile of GCamp6s) was performed. Peak responses to the first four odor stimulations were calculated by subtracting the mean DF/F in a $5 \mathrm{sec}$ window before the stimulus from the maximum value in the $20 \mathrm{sec}$ window after the stimulus. Interstimulus phases were extracted as $30 \mathrm{sec}$ intervals (10 seconds after a stimulus and 10 seconds before the next stimulus). Statistical analysis was performed with GraphPad Prism (version 9.1.2, GraphPad Software LLC).

\section{Chemical analysis of fly food}

\section{Chemicals}

The following compounds were obtained commercially from the sources given in parentheses: formic acid, sodium hydroxide (Merck, Darmstadt, Germany), histamine, ethanolamine, 2-phenylethylamine, putrescine, $\beta$-alanine, tyramine, spermine, spermidine, trichloroacetic acid (Sigma-Aldrich, Taufkirchen, Germany),

660 benzoyl chloride (VWR, Darmstadt, Germany), [2H4]-histamine, [2H4]-putrescine (CDN Isotopes, Quebec, Canada), [13C2]-ethanolamine, [13C3,15N]- $\beta$-alanine (Sigma-Aldrich, St. Louis, MO, USA), [2H4]phenylethylamine (Dr. Ehrenstorfer, Augsburg, Germany). The purity of all amines was checked by LC-MS and NMR experiments as described by Mayr and Schieberle (Mayr and Schieberle, 2012). Deuterated solvents were obtained from Euriso-Top (Gif-Sur-Yvette, France). Solvents used for HPLC-MS/MS

665 analysis were of LC-MS grade (Honeywell, Seelze, Germany); all other solvents were of HPLC grade (Merck Darmstadt, Germany). Water used for HPLC separation was purified by means of a Milli-Q water advantage A 10 water system (Millipore, Molsheim, France). [2H4]-Spermine, [2H2]-tyramine and [2H4]spermidine were synthesized and purified as reported earlier (Mayr and Schieberle, 2012). Standard flyfood consisted of the following ingredients: agar, corn flour, soy flour, yeast, malt, molasses, nipagin, ethanol,

\section{0 phosphoric acid.}

\section{Analysis of amines in fly food samples}

Quantification of biogenic amines and polyamines in flyfood was performed by means of a stable isotope dilution LC-MS/MS method after derivatization as already reported by Mayr and Schieberle (Mayr and Schieberle, 2012). Stock solutions. Stock solutions of the internal standards [13C3]-ethanolamine (52 $675 \mu \mathrm{g} / \mathrm{mL}),[13 \mathrm{C} 3,15 \mathrm{~N}]-\beta$-alanine $(36.0 \mu \mathrm{g} / \mathrm{mL}),[2 \mathrm{H} 4]$-histamine $(66.87 \mu \mathrm{g} / \mathrm{mL}),[2 \mathrm{H} 4]$-putrescine $(28.86$ $\mu \mathrm{g} / \mathrm{mL})$, [2H4]-spermine $(91.56 \mu \mathrm{g} / \mathrm{mL}),[2 \mathrm{H} 2]$-tyramine $(51 \mu \mathrm{g} / \mathrm{mL})$ and [2H4]-spermidine $(230 \mu \mathrm{g} / \mathrm{mL})$ and [2H4]-phenylethylamine $(109 \mu \mathrm{g} / \mathrm{mL})$ were prepared in aqueous tri-chloro-acetic acid $(10 \%)$ and stored at $7{ }^{\circ} \mathrm{C}$ until use.

\section{Sample workup}

680 Flyfood was frozen in liquid nitrogen and grounded in a mill (Moulinette, Moulinex, France). Aliquots (5 g) of each sample were spiked with an aliquot of the labled internal standards $([13 \mathrm{C} 3,15 \mathrm{~N}] \beta$-alanine $50 \mu \mathrm{L}$, [2H4]-histamine $100 \mu \mathrm{L}$, [2H4]-putrescine $100 \mu \mathrm{L}$, [2H4]-spermine $50 \mu \mathrm{L}$, [2H2]-tyramine $100 \mu \mathrm{L},[2 \mathrm{H} 4]$ spermidine $50 \mu \mathrm{L}$, and [2H4]-phenyl-ethylamine $109 \mu \mathrm{L})$, thereafter, aqueous tri-chloro-acetic acid (10\%, $40 \mathrm{~mL}$ ) was added, and vigorously stirred at room temperature. After an equilibration time of $30 \mathrm{~min}$, the

685 suspension was homogenated using an Ultraturrax (3 min, Jahnke and Kunkel, IKA-Labortechnik, Staufen im Breisgau, Germany), and ultrasonificated for another 10 minutes. The suspension obtained was centrifuged (10 $\mathrm{min}, 8000 \mathrm{rpm}$ ) and, finally, filtered (Schleicher \& Schuell filter). The $\mathrm{pH}$ of the filtrate was adjusted to 10 with aqueous sodium hydroxide $(1 \mathrm{M})$ and a solution of benzoyl chloride dissolved in 
acetonitrile $(30 \mathrm{~mL} ; 1 \mathrm{~g} / 250 \mathrm{~mL} \mathrm{ACN})$ was added. After stirring for $2 \mathrm{~h}$ at room temperature $\mathrm{pH}$ was adjusted to $\mathrm{pH}$ 2-3 using $\mathrm{HCl}$ (conc.). The benzamides were then extracted with dichloromethane ( $3 \times 20$ $\mathrm{mL}$ ), and the organic phases were combined, dried over $\mathrm{Na}_{2} \mathrm{SO}_{4}$, and evaporated to dryness at $30{ }^{\circ} \mathrm{C}$. The residue was dissolved in a mixture of acetonitrile and $0.1 \%$ aqueous formic acid $(20 / 80, \mathrm{v} / \mathrm{v})$ and filtered over a syringe filter ( $0.45 \mu \mathrm{m}$; Spartan 13/0.45 RC; Schleicher and Schuell, Dassel, Germany). The final filtrate was diluted with water $(1 / 20, \mathrm{v} / \mathrm{v})$. An aliquot $(10 \mu \mathrm{L})$ of the prepared sample was injected into the HPLC-MS/MS system.

High Performance Liquid Chromatography-Triple Quadrupole Mass Spectrometry (HPLC-MS/MS). HPLCMS/MS analysis was performed on a Surveyor high-performance chromatography system (Thermo Finnigan, Dreieich, Germany), equipped with a thermostated autosampler and a triple-quadrupol tandem mass spectrometer TQS Quantum Discovery (Thermo Electron, Dreieich, Germany). Temperature of the column compartment was set at $30{ }^{\circ} \mathrm{C}$ and autosampler temperature was $24{ }^{\circ} \mathrm{C}$. After sample injection $(10$ $\mu \mathrm{L})$, chromatography was carried out on a Synergy Fusion RP $\backslash$ SI $80 \AA$ column $(150$ x $2.0 \mathrm{~mm}$ id, $4 \mu \mathrm{m}$, Phenomenex, Aschaffenburg, Germany) using the following solvent gradient $(0.2 \mathrm{~mL} / \mathrm{min})$ of acetonitrile $(0.1 \%$ formic acid) as solvent $\mathrm{A}$ and formic acid ( $0.1 \%$ in water) as solvent $\mathrm{B}: 0 \mathrm{~min}, 0 \% \mathrm{~A} ; 1 \mathrm{~min}, 0 \% \mathrm{~A}$;

$7051.5 \mathrm{~min}, 35 \% \mathrm{~A} ; 20 \mathrm{~min}, 40 \%$ A; $26 \min , 50 \% \mathrm{~A} ; 27 \mathrm{~min}, 90 \% \mathrm{~A} ; 36 \mathrm{~min}, 90 \% \mathrm{~A} ; 37 \mathrm{~min}, 0 \% \mathrm{~A} . ; 52$ $\min , 0 \% \mathrm{~A}$. The mass spectrometer was operated in the positive electrospray ionization (ESI + ) mode, the spray needle voltage was set at $3.5 \mathrm{kV}$, the spray current at $5 \mu \mathrm{A}$, the temperature of the capillary was 300 , the capillary voltage at $35 \mathrm{~V}$. Nitrogen served as sheath and auxiliary gas, which was adjusted to 40 and 10 arbitary units. The collision cell was operated at a collision gas (argon) pressure of $0.13 \mathrm{~Pa}$. Mass transitions

710 of the pseudo molecular ions $([\mathrm{M}+\mathrm{H}]+)$ into specific product ions are summarized in Mayr and Schieberle (Mayr and Schieberle, 2012). Calibration curves for the calculation of the response factors and linear ranges of the analytes were measured as described before by Mayr and Schieberle (Mayr and Schieberle, 2012).

\section{Acknowledgments}

715 We thank our project students C. Biermeier, A. Erb, D. Mostert, V. Statovskii, K. Wald, R. Raab and D. Zapoglou for cross-validating T-maze experiments in independent experiments in single and multi-fly assays and extended research support for this project. We wish to acknowledge S. Aimon for helping us with whole brain imaging and imaging data analysis. We would especially like to thank Heidi MillerMommerskamp for technical support. Thanks also to A. Bates, M. Costa and G. Jefferis for discussions

720 regarding neurons of the lateral horn and access to EM tracing data. We acknowledge the Barnea, Jefferis and von Philipsborn labs for the provided fly lines. We are grateful to D. Neumeier and S. Kaviani-Nejad for technical assistance regarding the work on the polyamine concentration analysis in food. We would also like to thank N. Gompel, S. Sachse, K. Vogt and members of the Grunwald Kadow laboratory for comments on the manuscript and valuable feedback. This work was supported by the European Union (H2020, ERC

725 starting grant 'FlyContext' to IGK) and the German research foundation (SFB870, A04 to IGK, and GR4310/5-1).

\section{Author Contributions}

$\mathrm{AB}$ and IGK wrote the manuscript with help of all other authors. AB and IGK designed the study. AB conducted most of the single-fly experiments. JC conducted the MBON experiments at testing with Shibire $^{\text {ts } 1}$. MHL conducted the dTrpA1 at testing experiments, AB trained JC and MHL and cross-validated the experiments. AF and SH screened all lateral horn lines and cross-validated several mushroom body line experiments using T-maze assays. $\mathrm{AF}$ and $\mathrm{AB}$ carried out the immunohistochemistry experiments. $\mathrm{AF}$ 
performed all confocal imaging. SKP performed multiphoton calcium imaging experiments. PB performed and analyzed the whole brain lightfield imaging experiments. $\mathrm{CD}$ and $\mathrm{TH}$ provided the analysis of polyamine concentration in our fly food. All authors approved to the final version of the manuscript.

\section{Declaration of Interests}

The authors declare no competing interests.

\section{References}

Aimon, S., Katsuki, T., Jia, T., Grosenick, L., Broxton, M., Deisseroth, K., Sejnowski, T.J., and Greenspan, R.J. (2019). Fast near-whole-brain imaging in adult Drosophila during responses to stimuli and behavior.

745 PLoS Biol 17, e2006732. 10.1371/journal.pbio.2006732.

Aso, Y., Hattori, D., Yu, Y., Johnston, R.M., Iyer, N.A., Ngo, T.T., Dionne, H., Abbott, L.F., Axel, R., Tanimoto, H., and Rubin, G.M. (2014a). The neuronal architecture of the mushroom body provides a logic for associative learning. Elife 3, e04577. 10.7554/eLife.04577.

Aso, Y., Sitaraman, D., Ichinose, T., Kaun, K.R., Vogt, K., Belliart-Guerin, G., Placais, P.Y., Robie, A.A.,

750 Yamagata, N., Schnaitmann, C., et al. (2014b). Mushroom body output neurons encode valence and guide memory-based action selection in Drosophila. Elife 3, e04580. 10.7554/eLife.04580.

Azanchi, R., Kaun, K.R., and Heberlein, U. (2013). Competing dopamine neurons drive oviposition choice for ethanol in Drosophila. Proc Natl Acad Sci U S A 110, 21153-21158. 10.1073/pnas.1320208110.

Boswell, R.E., and Mahowald, A.P. (1985). tudor, a gene required for assembly of the germ plasm in

755 Drosophila melanogaster. Cell 43, 97-104. 10.1016/0092-8674(85)90015-7.

Bracker, L.B., Siju, K.P., Varela, N., Aso, Y., Zhang, M., Hein, I., Vasconcelos, M.L., and Grunwald Kadow, I.C. (2013). Essential role of the mushroom body in context-dependent CO(2) avoidance in Drosophila. Curr Biol 23, 1228-1234. 10.1016/j.cub.2013.05.029.

760 optics theory and 3-D deconvolution for the light field microscope. Optics express 21, 25418-25439. 10.1364/OE.21.025418.

Carlson, K.S., Gadziola, M.A., Dauster, E.S., and Wesson, D.W. (2018). Selective Attention Controls Olfactory Decisions and the Neural Encoding of Odors. Curr Biol 28, 2195-2205 e2194. 10.1016/j.cub.2018.05.011.

765 Carnell, S., Gibson, C., Benson, L., Ochner, C.N., and Geliebter, A. (2012). Neuroimaging and obesity: current knowledge and future directions. Obes Rev 13, 43-56. 10.1111/j.1467-789X.2011.00927.x.

Cernoch, J.M., and Porter, R.H. (1985). Recognition of maternal axillary odors by infants. Child development 56, 1593-1598.

Chapman, T., Bangham, J., Vinti, G., Seifried, B., Lung, O., Wolfner, M.F., Smith, H.K., and Partridge, L.

770 (2003). The sex peptide of Drosophila melanogaster: female post-mating responses analyzed by using RNA interference. Proc Natl Acad Sci U S A 100, 9923-9928. 10.1073/pnas.1631635100.

Chapman, T., and Wolfner, M.F. (2017). Reproductive behaviour: Make love, then war. Nat Ecol Evol 1, 174. 10.1038/s41559-017-0174.

Chin, S.G., Maguire, S.E., Huoviala, P., Jefferis, G., and Potter, C.J. (2018). Olfactory Neurons and Brain

775 Centers Directing Oviposition Decisions in Drosophila. Cell Rep 24, 1667-1678. 10.1016/j.celrep.2018.07.018.

Chow, C.Y., Avila, F.W., Clark, A.G., and Wolfner, M.F. (2015). Induction of excessive endoplasmic reticulum stress in the Drosophila male accessory gland results in infertility. PLoS One 10, e0119386. 10.1371/journal.pone.0119386.

780 Chu, B., Chui, V., Mann, K., and Gordon, M.D. (2014). Presynaptic gain control drives sweet and bitter taste integration in Drosophila. Curr Biol 24, 1978-1984. 10.1016/j.cub.2014.07.020.

Cohn, R., Morantte, I., and Ruta, V. (2015). Coordinated and Compartmentalized Neuromodulation Shapes Sensory Processing in Drosophila. Cell 163, 1742-1755. 10.1016/j.cell.2015.11.019.

Cury, K.M., Prud'homme, B., and Gompel, N. (2019). A short guide to insect oviposition: when, where and 
how to lay an egg. J Neurogenet 33, 75-89. 10.1080/01677063.2019.1586898.

Das, S., Trona, F., Khallaf, M.A., Schuh, E., Knaden, M., Hansson, B.S., and Sachse, S. (2017). Electrical synapses mediate synergism between pheromone and food odors in Drosophila melanogaster. Proc Natl Acad Sci U S A 114, E9962-E9971. 10.1073/pnas.1712706114.

Dey, S., Chamero, P., Pru, J.K., Chien, M.S., Ibarra-Soria, X., Spencer, K.R., Logan, D.W., Matsunami, H., Peluso, J.J., and Stowers, L. (2015). Cyclic Regulation of Sensory Perception by a Female Hormone Alters Behavior. Cell 161, 1334-1344. 10.1016/j.cell.2015.04.052.

Dickson, B.J. (2008). Wired for sex: the neurobiology of Drosophila mating decisions. Science 322, 904-909. 322/5903/904 [pii]

10.1126/science.1159276.

795 Dolan, M.J., Belliart-Guerin, G., Bates, A.S., Frechter, S., Lampin-Saint-Amaux, A., Aso, Y., Roberts, R.J.V., Schlegel, P., Wong, A., Hammad, A., et al. (2018). Communication from Learned to Innate Olfactory Processing Centers Is Required for Memory Retrieval in Drosophila. Neuron 100, 651-668 e658. 10.1016/j.neuron.2018.08.037.

Dolan, M.J., Frechter, S., Bates, A.S., Dan, C., Huoviala, P., Roberts, R.J., Schlegel, P., Dhawan, S., Tabano, 800 R., Dionne, H., et al. (2019). Neurogenetic dissection of the Drosophila lateral horn reveals major outputs, diverse behavioural functions, and interactions with the mushroom body. Elife 8. 10.7554/eLife.43079.

Duménil, C., Woud, D., Pinto, F., Alkema, J.T., Jansen, I., Van Der Geest, A.M., Roessingh, S., and Billeter, J.C. (2016). Pheromonal Cues Deposited by Mated Females Convey Social Information about Egg-Laying Sites in Drosophila Melanogaster. J Chem Ecol 42, 259-269. 10.1007/s10886-016-0681-3.

805 Ebrahim, S.A., Dweck, H.K., Stokl, J., Hofferberth, J.E., Trona, F., Weniger, K., Rybak, J., Seki, Y., Stensmyr, M.C., Sachse, S., et al. (2015). Drosophila Avoids Parasitoids by Sensing Their Semiochemicals via a Dedicated Olfactory Circuit. PLoS Biol 13, e1002318. 10.1371/journal.pbio.1002318.

Ejima, A., Smith, B.P., Lucas, C., van der Goes van Naters, W., Miller, C.J., Carlson, J.R., Levine, J.D., and Griffith, L.C. (2007). Generalization of courtship learning in Drosophila is mediated by cis-vaccenyl acetate.

810 Curr Biol 17, 599-605. 10.1016/j.cub.2007.01.053.

Frechter, S., Bates, A.S., Tootoonian, S., Dolan, M.J., Manton, J.D., Jamasb, A.R., Kohl, J., Bock, D., and Jefferis, G.S. (2019). Functional and anatomical specificity in a higher olfactory centre. Elife 8. 10.7554/eLife.44590.

Galbally, M., Lewis, A.J., Ijzendoorn, M., and Permezel, M. (2011). The role of oxytocin in mother-infant 815 relations: a systematic review of human studies. Harv Rev Psychiatry 19, 1-14. 10.3109/10673229.2011.549771.

Gervasi, N., Tchenio, P., and Preat, T. (2010). PKA dynamics in a Drosophila learning center: coincidence detection by rutabaga adenylyl cyclase and spatial regulation by dunce phosphodiesterase. Neuron $65,516-$ 529. 10.1016/j.neuron.2010.01.014.

820 Gou, B., Liu, Y., Guntur, A.R., Stern, U., and Yang, C.H. (2014). Mechanosensitive neurons on the internal reproductive tract contribute to egg-laying-induced acetic acid attraction in Drosophila. Cell Rep 9, 522-530. 10.1016/j.celrep.2014.09.033.

Griffith, L.C., and Ejima, A. (2009). Courtship learning in Drosophila melanogaster: diverse plasticity of a reproductive behavior. Learn Mem 16, 743-750. 16/12/743 [pii]

$825 \quad 10.1101 / 1 \mathrm{~lm} .956309$.

Grosjean, Y., Rytz, R., Farine, J.P., Abuin, L., Cortot, J., Jefferis, G.S., and Benton, R. (2011). An olfactory receptor for food-derived odours promotes male courtship in Drosophila. Nature 478, 236-240. 10.1038/nature10428.

Grunwald Kadow, I.C. (2018). State-dependent plasticity of innate behavior in fruit flies. Curr Opin Neurobiol $830 \quad 54,60-65.10 .1016 /$ j.conb.2018.08.014.

Hadjieconomou, D., King, G., Gaspar, P., Mineo, A., Blackie, L., Ameku, T., Studd, C., de Mendoza, A., Diao, F., White, B.H., et al. (2020). Enteric neurons increase maternal food intake during reproduction. Nature 587, 455-459. 10.1038/s41586-020-2866-8.

Heisenberg, M. (2003). Mushroom body memoir: from maps to models. Nat Rev Neurosci 4, 266-275.

$83510.1038 / \mathrm{nrn} 1074$

nrn1074 [pii]. 
Hoekzema, E., Barba-Muller, E., Pozzobon, C., Picado, M., Lucco, F., Garcia-Garcia, D., Soliva, J.C., Tobena, A., Desco, M., Crone, E.A., et al. (2017). Pregnancy leads to long-lasting changes in human brain structure. Nat Neurosci 20, 287-296. 10.1038/nn.4458.

Hussain, A., Ucpunar, H.K., Zhang, M., Loschek, L.F., and Grunwald Kadow, I.C. (2016a). Neuropeptides Modulate Female Chemosensory Processing upon Mating in Drosophila. PLoS Biol 14, e1002455. 10.1371/journal.pbio.1002455.

Hussain, A., Zhang, M., Ucpunar, H.K., Svensson, T., Quillery, E., Gompel, N., Ignell, R., and Grunwald Kadow, I.C. (2016b). Ionotropic Chemosensory Receptors Mediate the Taste and Smell of Polyamines. PLoS 845 Biol 14, e1002454. 10.1371/journal.pbio.1002454.

Insel, T.R., Preston, S., and Winslow, J.T. (1995). Mating in the monogamous male: behavioral consequences. Physiol Behav 57, 615-627.

Ito, K., Shinomiya, K., Ito, M., Armstrong, J.D., Boyan, G., Hartenstein, V., Harzsch, S., Heisenberg, M., Homberg, U., Jenett, A., et al. (2014). A systematic nomenclature for the insect brain. Neuron 81, 755-765.

$850 \quad 10.1016 /$ j.neuron.2013.12.017.

Jeanne, J.M., Fisek, M., and Wilson, R.I. (2018). The Organization of Projections from Olfactory Glomeruli onto Higher-Order Neurons. Neuron 98, 1198-1213 e1196. 10.1016/j.neuron.2018.05.011.

Jefferis, G.S., Potter, C.J., Chan, A.M., Marin, E.C., Rohlfing, T., Maurer, C.R., Jr., and Luo, L. (2007). Comprehensive maps of Drosophila higher olfactory centers: spatially segregated fruit and pheromone 855 representation. Cell 128, 1187-1203. S0092-8674(07)00204-8 [pii]

10.1016/j.cell.2007.01.040.

Kalac, P. (2014). Health effects and occurrence of dietary polyamines: a review for the period 2005-mid 2013. Food chemistry 161, 27-39. 10.1016/j.foodchem.2014.03.102.

Keleman, K., Vrontou, E., Kruttner, S., Yu, J.Y., Kurtovic-Kozaric, A., and Dickson, B.J. (2012). Dopamine 860 neurons modulate pheromone responses in Drosophila courtship learning. Nature 489, 145-149. 10.1038/nature11345.

Kim, Y.J., Bartalska, K., Audsley, N., Yamanaka, N., Yapici, N., Lee, J.Y., Kim, Y.C., Markovic, M., Isaac, E., Tanaka, Y., and Dickson, B.J. (2010). MIPs are ancestral ligands for the sex peptide receptor. Proc Natl Acad Sci U S A 107, 6520-6525. 10.1073/pnas.0914764107.

865 Kirkhart, C., and Scott, K. (2015). Gustatory learning and processing in the Drosophila mushroom bodies. J Neurosci 35, 5950-5958. 10.1523/JNEUROSCI.3930-14.2015.

Kitamoto, T. (2002). Targeted expression of temperature-sensitive dynamin to study neural mechanisms of complex behavior in Drosophila. J Neurogenet 16, 205-228. VX8G3E00EPMLBACJ [pii].

Koch, M., and Ehret, G. (1989). Estradiol and parental experience, but not prolactin are necessary for 870 ultrasound recognition and pup-retrieving in the mouse. Physiol Behav 45, 771-776.

Kohl, J., Ostrovsky, A.D., Frechter, S., and Jefferis, G.S. (2013). A bidirectional circuit switch reroutes pheromone signals in male and female brains. Cell 155, 1610-1623. 10.1016/j.cell.2013.11.025.

Krashes, M.J., DasGupta, S., Vreede, A., White, B., Armstrong, J.D., and Waddell, S. (2009). A neural circuit mechanism integrating motivational state with memory expression in Drosophila. Cell 139, 416-427.

875 10.1016/j.cell.2009.08.035.

Kubli, E. (2003). Sex-peptides: seminal peptides of the Drosophila male. Cell Mol Life Sci 60, 1689-1704. 10.1007/s00018-003-3052.

Laturney, M., and Billeter, J.C. (2016). Drosophila melanogaster females restore their attractiveness after mating by removing male anti-aphrodisiac pheromones. Nat Commun 7, 12322. 10.1038/ncomms 12322.

880 Lebreton, S., Trona, F., Borrero-Echeverry, F., Bilz, F., Grabe, V., Becher, P.G., Carlsson, M.A., Nassel, D.R., Hansson, B.S., Sachse, S., and Witzgall, P. (2015). Feeding regulates sex pheromone attraction and courtship in Drosophila females. Sci Rep 5, 13132. 10.1038/srep13132.

LeDue, E.E., Mann, K., Koch, E., Chu, B., Dakin, R., and Gordon, M.D. (2016). Starvation-Induced Depotentiation of Bitter Taste in Drosophila. Curr Biol 26, 2854-2861. 10.1016/j.cub.2016.08.028.

885 Leinwand, S.G., and Chalasani, S.H. (2011). Olfactory networks: from sensation to perception. Curr Opin Genet Dev 21, 806-811. 10.1016/j.gde.2011.07.006.

Leinwand, S.G., and Scott, K. (2021). Juvenile hormone drives the maturation of spontaneous mushroom body neural activity and learned behavior. Neuron 109, 1836-1847 e1835. 10.1016/j.neuron.2021.04.006. 
Lewis, L.P., Siju, K.P., Aso, Y., Friedrich, A.B., Bulteel, A.J., Rubin, G.M., and Grunwald Kadow, I.C. (2015). A Higher Brain Circuit for Immediate Integration of Conflicting Sensory Information in Drosophila. Curr Biol 25, 2203-2214. 10.1016/j.cub.2015.07.015.

Li, F., Lindsey, J., Marin, E.C., Otto, N., Dreher, M., Dempsey, G., Stark, I., Bates, A.S., Pleijzier, M.W., Schlegel, P., et al. (2020). The connectome of the adult $<$ em $>$ Drosophila $</$ em $>$ mushroom body: implications for function. bioRxiv, 2020.2008.2029.273276. 10.1101/2020.08.29.273276.

895 Lin, C.C., Prokop-Prigge, K.A., Preti, G., and Potter, C.J. (2015). Food odors trigger Drosophila males to deposit a pheromone that guides aggregation and female oviposition decisions. Elife 4. 10.7554/eLife.08688. Livingstone, M.S., Sziber, P.P., and Quinn, W.G. (1984). Loss of calcium/calmodulin responsiveness in adenylate cyclase of rutabaga, a Drosophila learning mutant. Cell 37, 205-215. 10.1016/0092-8674(84)903167.

900 Louis, T., Stahl, A., Boto, T., and Tomchik, S.M. (2018). Cyclic AMP-dependent plasticity underlies rapid changes in odor coding associated with reward learning. Proc Natl Acad Sci U S A 115, E448-E457. 10.1073/pnas.1709037115.

Marlin, B.J., Mitre, M., D'Amour J, A., Chao, M.V., and Froemke, R.C. (2015). Oxytocin enables maternal behaviour by balancing cortical inhibition. Nature 520, 499-504. 10.1038/nature14402.

905 Mayr, C.M., and Schieberle, P. (2012). Development of stable isotope dilution assays for the simultaneous quantitation of biogenic amines and polyamines in foods by LC-MS/MS. J Agric Food Chem 60, 3026-3032. $10.1021 / j f 204900 \mathrm{v}$.

Miller-Fleming, L., Olin-Sandoval, V., Campbell, K., and Ralser, M. (2015). Remaining Mysteries of Molecular Biology: The Role of Polyamines in the Cell. J Mol Biol 427, 3389-3406.

$910 \quad$ 10.1016/j.jmb.2015.06.020.

Min, S., Ai, M., Shin, S.A., and Suh, G.S. (2013). Dedicated olfactory neurons mediating attraction behavior to ammonia and amines in Drosophila. Proc Natl Acad Sci U S A 110, E1321-1329. 10.1073/pnas.1215680110.

Montague, S.A., and Baker, B.S. (2016). Memory Elicited by Courtship Conditioning Requires Mushroom

915 Body Neuronal Subsets Similar to Those Utilized in Appetitive Memory. PLoS One 11, e0164516. 10.1371/journal.pone.0164516.

Moshitzky, P., Fleischmann, I., Chaimov, N., Saudan, P., Klauser, S., Kubli, E., and Applebaum, S.W. (1996). Sex-peptide activates juvenile hormone biosynthesis in the Drosophila melanogaster corpus allatum. Arch Insect Biochem Physiol 32, 363-374. 10.1002/(SICI)1520-6327(1996)32:3/4<363::AID-ARCH9>3.0.CO;2-

$920 \mathrm{~T}$.

Ochsenbein-Kolble, N., von Mering, R., Zimmermann, R., and Hummel, T. (2007). Changes in olfactory function in pregnancy and postpartum. International journal of gynaecology and obstetrics: the official organ of the International Federation of Gynaecology and Obstetrics 97, 10-14. 10.1016/j.ijgo.2006.12.009.

Owald, D., Felsenberg, J., Talbot, C.B., Das, G., Perisse, E., Huetteroth, W., and Waddell, S. (2015). Activity 925 of defined mushroom body output neurons underlies learned olfactory behavior in Drosophila. Neuron 86 , 417-427. 10.1016/j.neuron.2015.03.025.

Palouzier-Paulignan, B., Lacroix, M.C., Aime, P., Baly, C., Caillol, M., Congar, P., Julliard, A.K., Tucker, K., and Fadool, D.A. (2012). Olfaction under metabolic influences. Chem Senses 37, 769-797. 10.1093/chemse/bjs059.

930 Piper, M.D., Blanc, E., Leitão-Gonçalves, R., Yang, M., He, X., Linford, N.J., Hoddinott, M.P., Hopfen, C., Soultoukis, G.A., Niemeyer, C., et al. (2014). A holidic medium for Drosophila melanogaster. Nat Methods 11, 100-105. 10.1038/nmeth.2731.

Reisenman, C.E., Riffell, J.A., and Hildebrand, J.G. (2009). Neuroethology of oviposition behavior in the moth Manduca sexta. Ann N Y Acad Sci 1170, 462-467. 10.1111/j.1749-6632.2009.03875.x.

935 Ribeiro, C., and Dickson, B.J. (2010). Sex peptide receptor and neuronal TOR/S6K signaling modulate nutrient balancing in Drosophila. Curr Biol 20, 1000-1005. 10.1016/j.cub.2010.03.061.

Rolls, E.T. (2007). Sensory processing in the brain related to the control of food intake. Proc Nutr Soc 66, 96112. $10.1017 / \mathrm{S} 0029665107005332$.

Root, C.M., Ko, K.I., Jafari, A., and Wang, J.W. (2011). Presynaptic facilitation by neuropeptide signaling mediates odor-driven food search. Cell 145, 133-144. S0092-8674(11)00122-X [pii] 
10.1016/j.cell.2011.02.008.

Rosenzweig, M., Kang, K., and Garrity, P.A. (2008). Distinct TRP channels are required for warm and cool avoidance in Drosophila melanogaster. Proc Natl Acad Sci U S A 105, 14668-14673. 0805041105 [pii] 10.1073/pnas.0805041105.

45 Ruta, V., Datta, S.R., Vasconcelos, M.L., Freeland, J., Looger, L.L., and Axel, R. (2010). A dimorphic pheromone circuit in Drosophila from sensory input to descending output. Nature 468, 686-690. 10.1038/nature09554.

Sayin, S., Boehm, A.C., Kobler, J.M., De Backer, J.F., and Grunwald Kadow, I.C. (2018). Internal State Dependent Odor Processing and Perception-The Role of Neuromodulation in the Fly Olfactory System. Front 950 Cell Neurosci 12, 11. 10.3389/fncel.2018.00011.

Sayin, S., De Backer, J.F., Siju, K.P., Wosniack, M.E., Lewis, L.P., Frisch, L.M., Gansen, B., Schlegel, P., Edmondson-Stait, A., Sharifi, N., et al. (2019). A Neural Circuit Arbitrates between Persistence and Withdrawal in Hungry Drosophila. Neuron. 10.1016/j.neuron.2019.07.028.

Scheffer, L.K., Xu, C.S., Januszewski, M., Lu, Z., Takemura, S.Y., Hayworth, K.J., Huang, G.B., Shinomiya,

955 K., Maitlin-Shepard, J., Berg, S., et al. (2020). A connectome and analysis of the adult. Elife 9. 10.7554/eLife.57443.

Scheunemann, L., Lampin-Saint-Amaux, A., Schor, J., and Preat, T. (2019). A sperm peptide enhances longterm memory in female Drosophila. Sci Adv 5, eaax3432. 10.1126/sciadv.aax3432.

Schindelin, J., Arganda-Carreras, I., Frise, E., Kaynig, V., Longair, M., Pietzsch, T., Preibisch, S., Rueden,

960 C., Saalfeld, S., Schmid, B., et al. (2012). Fiji: an open-source platform for biological-image analysis. Nat Methods 9, 676-682. 10.1038/nmeth.2019.

Sejourne, J., Placais, P.Y., Aso, Y., Siwanowicz, I., Trannoy, S., Thoma, V., Tedjakumala, S.R., Rubin, G.M., Tchenio, P., Ito, K., et al. (2011). Mushroom body efferent neurons responsible for aversive olfactory memory retrieval in Drosophila. Nat Neurosci 14, 903-910. 10.1038/nn.2846

965 nn.2846 [pii].

Shao, L., Chung, P., Wong, A., Siwanowicz, I., Kent, C.F., Long, X., and Heberlein, U. (2019). A Neural Circuit Encoding the Experience of Copulation in Female Drosophila. Neuron 102, 1025-1036 e1026. 10.1016/j.neuron.2019.04.009.

Siegel, R.W., and Hall, J.C. (1979). Conditioned responses in courtship behavior of normal and mutant 970 Drosophila. Proc Natl Acad Sci U S A 76, 3430-3434.

Siju, K.P., Štih, V., Aimon, S., Gjorgjieva, J., Portugues, R., and Grunwald Kadow, I.C. (2020). Valence and State-Dependent Population Coding in Dopaminergic Neurons in the Fly Mushroom Body. Curr Biol 30, 2104-2115.e2104. 10.1016/j.cub.2020.04.037.

Silbering, A.F., Rytz, R., Grosjean, Y., Abuin, L., Ramdya, P., Jefferis, G.S., and Benton, R. (2011).

975 Complementary function and integrated wiring of the evolutionarily distinct Drosophila olfactory subsystems. J Neurosci 31, 13357-13375. 10.1523/JNEUROSCI.2360-11.2011.

Strutz, A., Soelter, J., Baschwitz, A., Farhan, A., Grabe, V., Rybak, J., Knaden, M., Schmuker, M., Hansson, B.S., and Sachse, S. (2014). Decoding odor quality and intensity in the Drosophila brain. Elife 3, e04147. 10.7554/eLife.04147.

980 Sullivan, R.M. (2003). Developing a sense of safety: the neurobiology of neonatal attachment. Ann N Y Acad Sci $1008,122-131$.

Talay, M., Richman, E.B., Snell, N.J., Hartmann, G.G., Fisher, J.D., Sorkac, A., Santoyo, J.F., Chou-Freed, C., Nair, N., Johnson, M., et al. (2017). Transsynaptic Mapping of Second-Order Taste Neurons in Flies by trans-Tango. Neuron 96, 783-795 e784. 10.1016/j.neuron.2017.10.011.

985 Tsao, C.H., Chen, C.C., Lin, C.H., Yang, H.Y., and Lin, S. (2018). Drosophila mushroom bodies integrate hunger and satiety signals to control innate food-seeking behavior. Elife 7. 10.7554/eLife.35264.

Varela, N., Gaspar, M., Dias, S., and Vasconcelos, M.L. (2019). Avoidance response to CO2 in the lateral horn. PLoS Biol 17, e2006749. 10.1371/journal.pbio.2006749.

Walker, S.J., Corrales-Carvajal, V.M., and Ribeiro, C. (2015). Postmating Circuitry Modulates Salt Taste

990 Processing to Increase Reproductive Output in Drosophila. Curr Biol. 10.1016/j.cub.2015.08.043.

Woller, A., Bandow, P., Aimon, S., and Grunwald Kadow, I.C. (2021). Preparing Adult Drosophila melanogaster for Whole Brain Imaging during Behavior and Stimuli Responses. J Vis Exp. 10.3791/61876. 
Yapici, N., Kim, Y.J., Ribeiro, C., and Dickson, B.J. (2008). A receptor that mediates the post-mating switch in Drosophila reproductive behaviour. Nature 451,33-37. 10.1038/nature06483.

995 Zhao, M., Garland, T., Jr., Chappell, M.A., Andrew, J.R., and Saltzman, W. (2017). Metabolic and affective consequences of fatherhood in male California mice. Physiol Behav 177, 57-67. 10.1016/j.physbeh.2017.04.010.

Zhao, X., Lenek, D., Dag, U., Dickson, B.J., and Keleman, K. (2018). Persistent activity in a recurrent circuit underlies courtship memory in Drosophila. Elife 7. 10.7554/eLife.31425.

1000 Zheng, Z., Lauritzen, J.S., Perlman, E., Robinson, C.G., Nichols, M., Milkie, D., Torrens, O., Price, J., Fisher, C.B., Sharifi, N., et al. (2018). A Complete Electron Microscopy Volume of the Brain of Adult Drosophila melanogaster. Cell 174, 730-743 e722. 10.1016/j.cell.2018.06.019. 
Figures and Figure legends
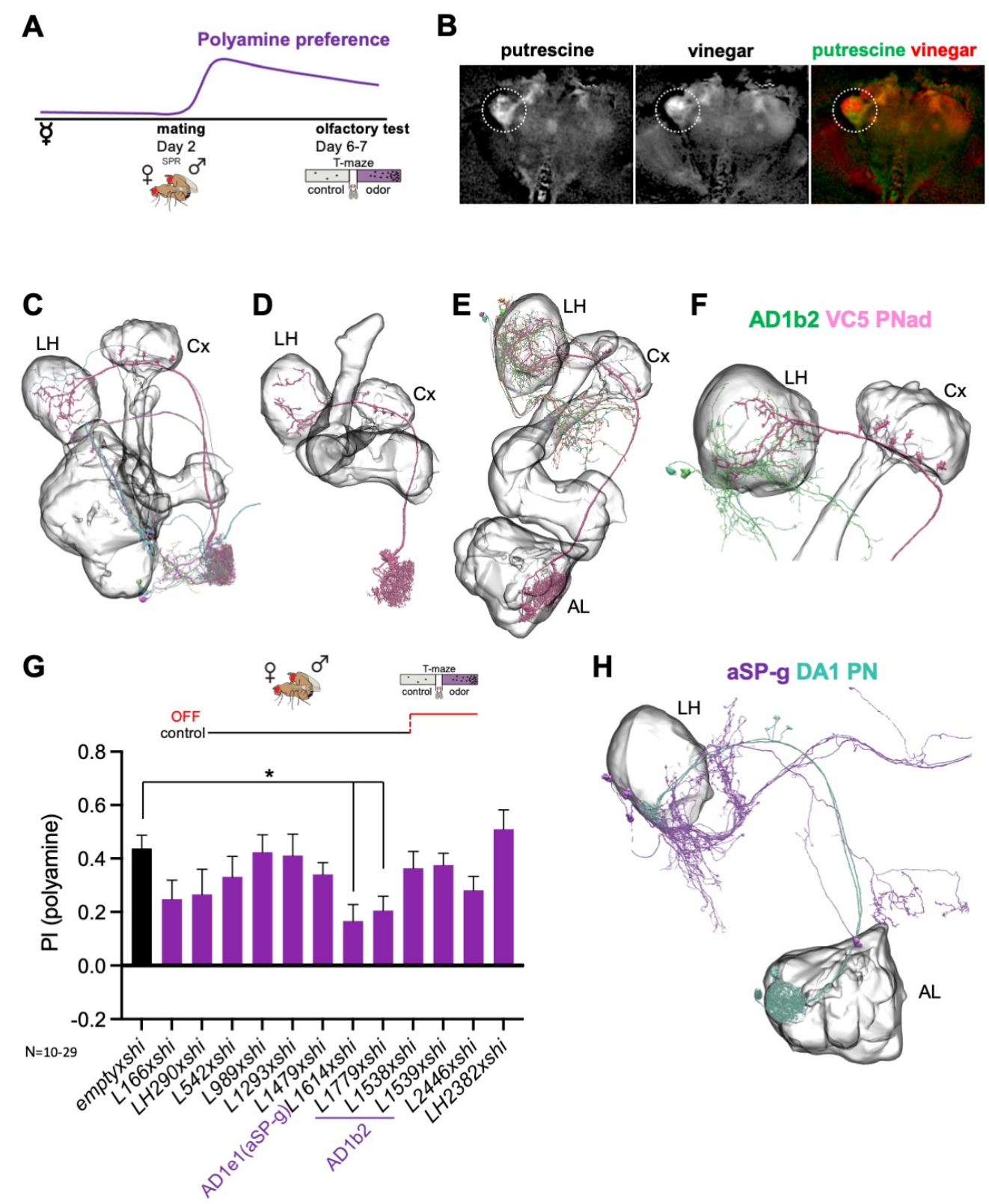

Figure 1. Lateral horn pathway contributes to polyamine attraction.

(A) Scheme depicting paradigm used in all experiments. Virgin females were kept alone for 6-7 days or mated on day 2 for $24 \mathrm{~h}$, then separated, and test at day 6-7 for their preference for the odor of the polyamine, putrescine. Mated females show a high preference for polyamine, which depends on matinginduced modulation of polyamine-detecting olfactory neurons (see text for details). (B) In vivo whole brain lightfield calcium imaging upon stimulation with putrescine or vinegar (nsyb-Gal4; UAS-GCaMP6m). Putrescine and vinegar activate different areas in the lateral horn (LH, dotted line). (C) Electron microscopy (EM)-based reconstruction showing projection neurons (PN) innervating the VC5 glomerulus. (D) VC5

1015 PNad (pink) innervates the LH and the mushroom body (MB). (E) VC5 PNad provides input to different LH neurons. (F) LH output neuron (LHON) AD1b2 is the main downstream partner of VC5 PNad. (G) T-maze behavioral screen at restrictive temperature at test $\left(32^{\circ} \mathrm{C}\right)$ of candidate LHONs in mated females reveals that 
two LHON types are required for full attraction to putrescine. 'emptyxshi' represents the control group and stands for $p B D P G A L 4 ; U A S$-shits , the Gal4 vector used to generate all MB-Gal4 lines without an expression driving enhancer. $(\mathrm{H})$ LHON aSP-g receives input from the DA1 PN. Note that the DA glomerulus is responds to the pheromone cVA. One-way Anova, Bonferroni corrected; P-values: *: $<0.05$. LH: Lateral horn, Cx: Mushroom body calyx, AL: Antennal lobe

A

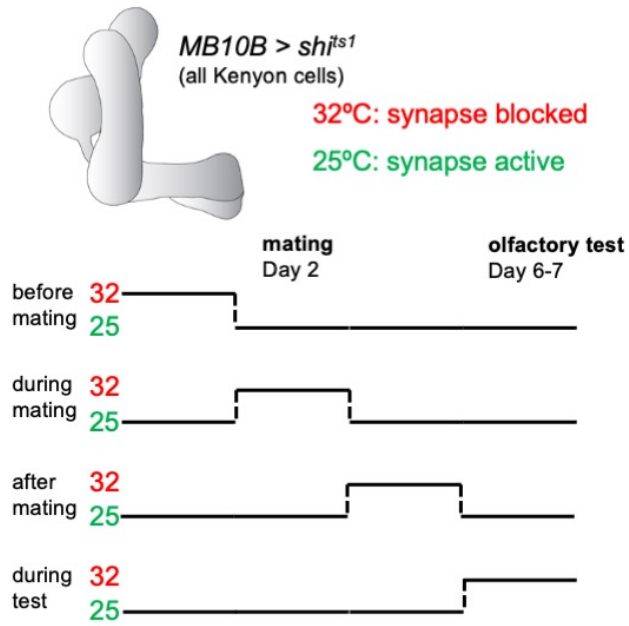

C

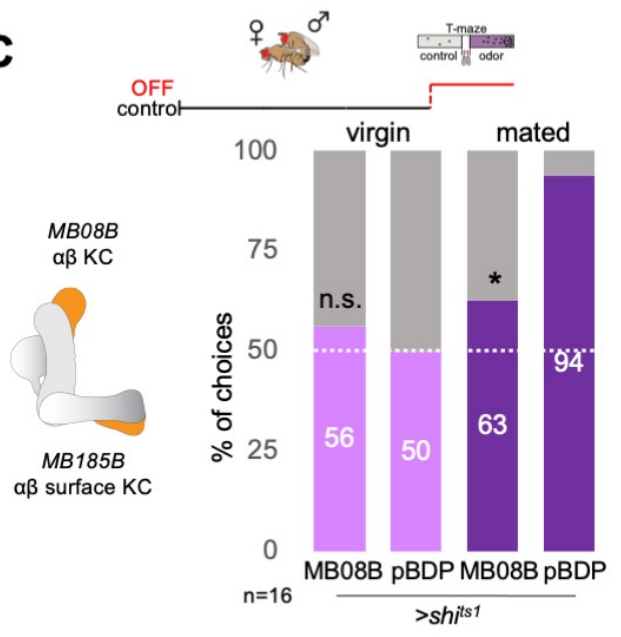

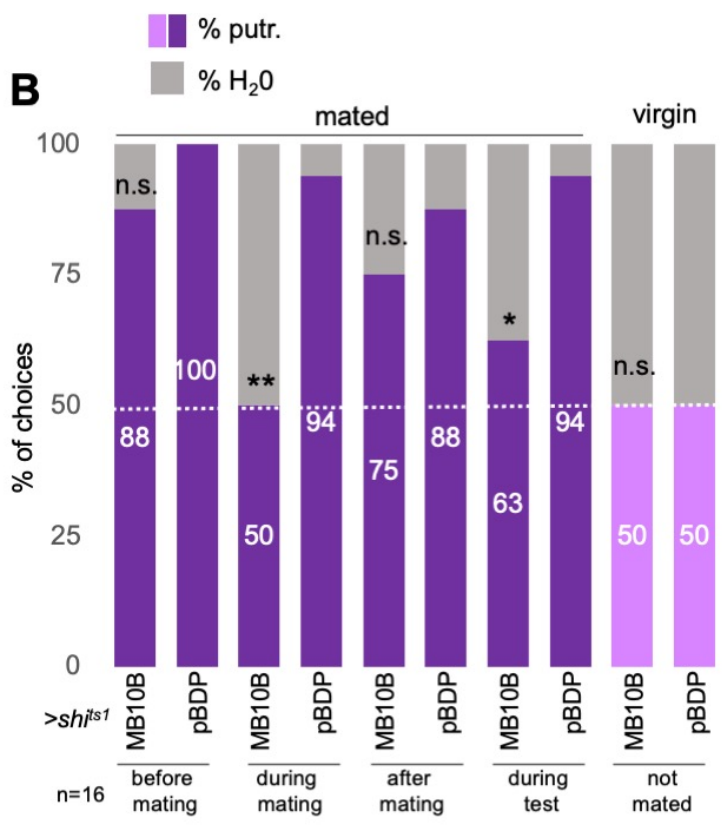

D

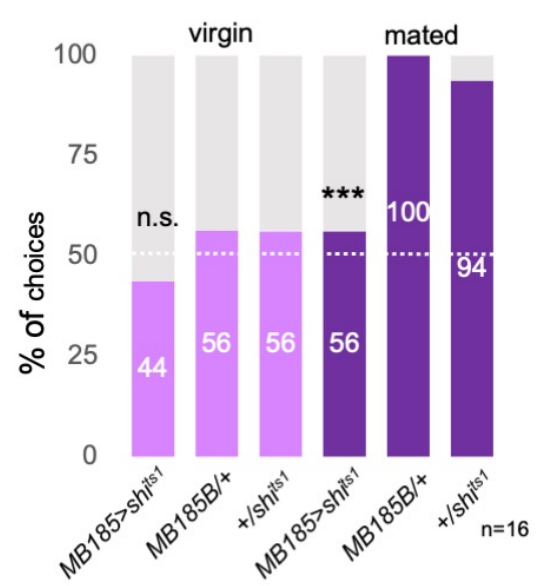

Figure 2. The mushroom body and learning mechanisms are required in long-term preference change upon mating.

(A) Scheme of experimental paradigm used to block Kenyon cell (KC) synaptic output at precise times during the experiment. (B) KC synaptic output is required during mating and during preference test. Single female preference for putrescine in T-maze assay. (C) Inhibition of $\alpha \beta$-type KC output (MB08B-Gal4;UAS$\left.s h i^{i s I}\right)$ at the time of olfactory preference test significantly reduced mated female preference for putrescine. Single female preference for putrescine in T-maze assay. 50\% represents chance. Fisher's exact test. Pvalues: $n$.s. $:>0.1, *:<0.1, * *:<0.05, * * *:<0.001$. (D) Inhibition of $\alpha \beta$ surface-type $\mathrm{KC}$ output (MB185BGal4;UAS-shits $)$ at the time of olfactory preference test also significantly reduced mated female preference 
for putrescine. Single female preference for putrescine in T-maze assay. 50\% represents chance. Depicted Pvalues, unless indicated otherwise, refer to the difference between experimental and control group in virgins or mated females, respectively.
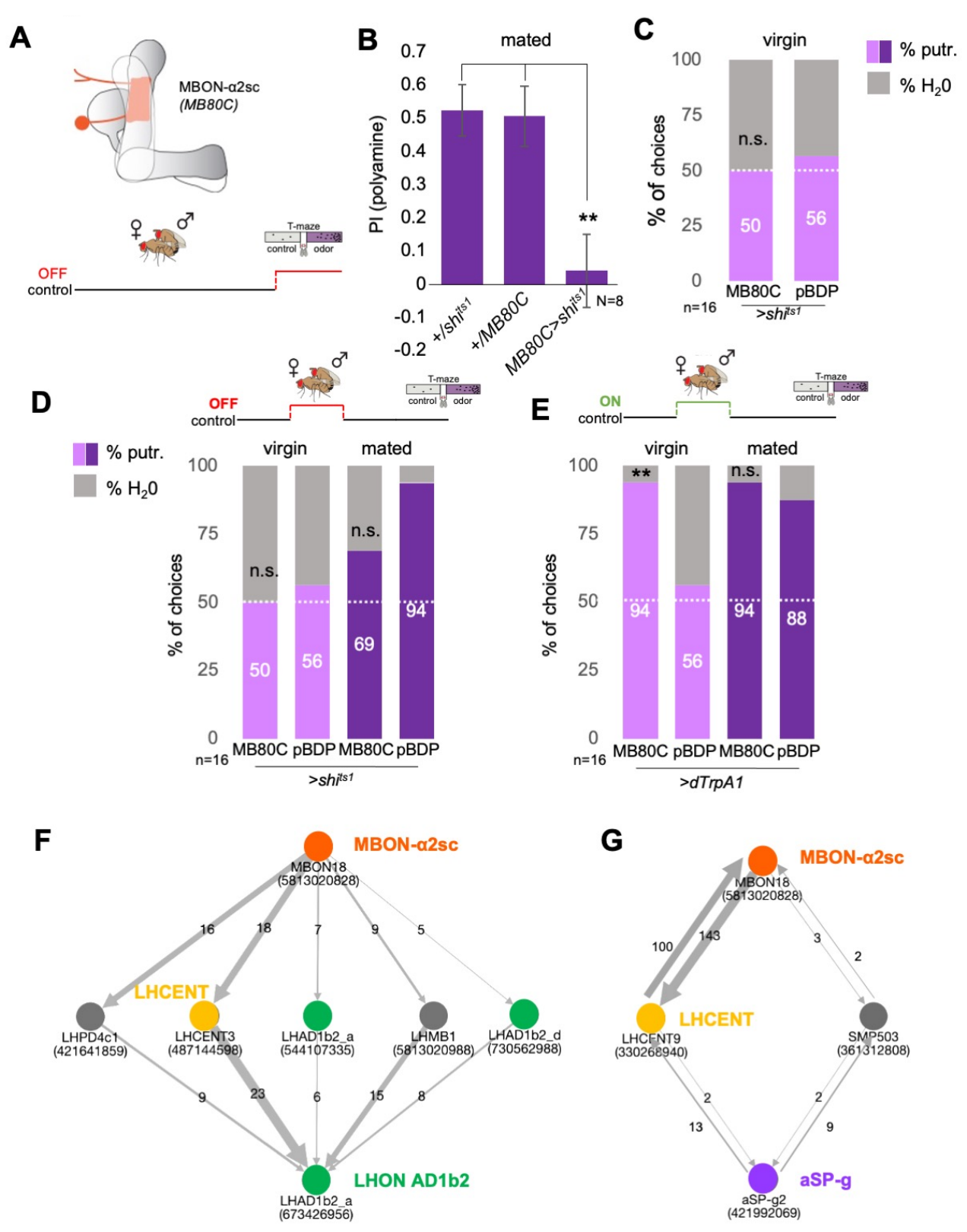

1040 Figure 3. A role for a lateral horn-innervating MBON in mating state-dependent odor attraction.

(A) top, scheme of MBON- $\alpha 2 \mathrm{sc}$ and expression of line MB80C. bottom, scheme showing experimental paradigm. Neuronal output was blocked at the time of preference test. (B) Inhibition of MBON- $\alpha 2 s c$ output (MB80C-Gal4;UAS-shits $)$ at the time of test significantly reduced mated female preference for putrescine. T-maze group assay. Bars depict preference index (PI) \pm SEM. Student's t-test; $* *<0.01$ (C) Inhibition of

$1045 \mathrm{MBON}-\alpha 2 \mathrm{sc}$ output (MB80C-Gal4;UAS-shit $\left.{ }^{\text {tsI }}\right)$ at the time of test had no effect on virgin female behavior. Single female preference for putrescine in T-maze assay. 50\% represents chance. (D) Inhibition of MBONa2sc output (MB80C-Gal4;UAS-shi ${ }^{\text {tsI }}$ ) during mating did not significantly reduce mated female preference 
for putrescine. Single female preference for putrescine in T-maze assay. 50\% represents chance. (E) Activation of MBON- $\alpha 2 \mathrm{sc}$ output (MB80C-Gal4;UAS-dTrpA1) instead of mating was sufficient to strongly enhance virgin female preference for putrescine. Single female preference for putrescine in T-maze assay. $50 \%$ represents chance. Fisher's exact test. P-values: n.s.: $>0.1, *:<0.1, * *:<0.05, * * *:<0.001$. (F) MBON$\alpha 2 s c$ directly and indirectly innervates LHON AD1b2. (G) MBON- $\alpha 2 s c$ forms bidirectional indirect synaptic connections with aSP-g.

A
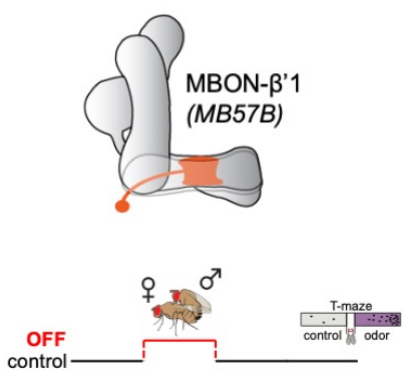

C
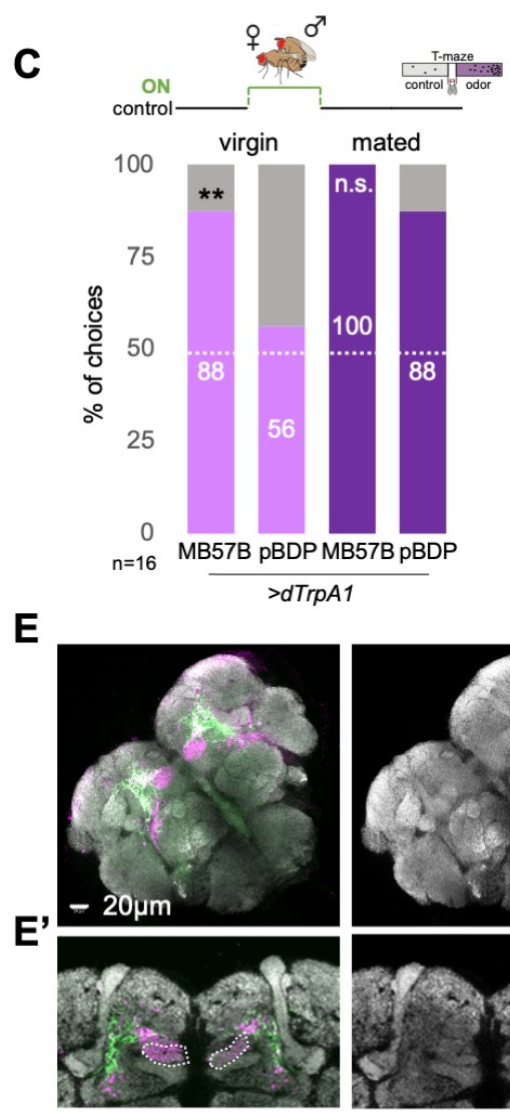

E'

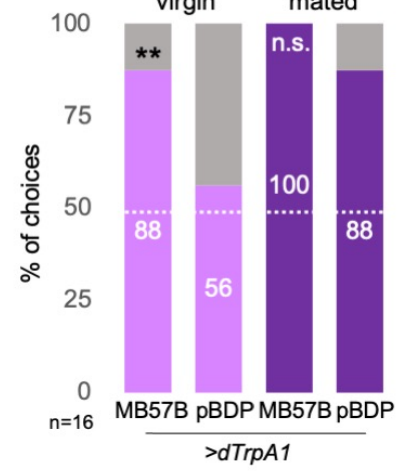

merge
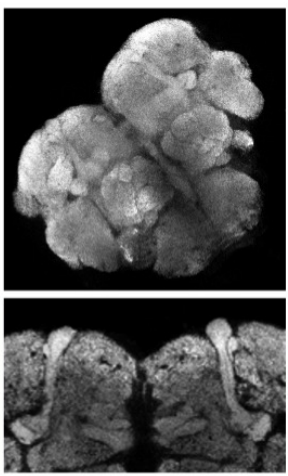

a-Ncad
B

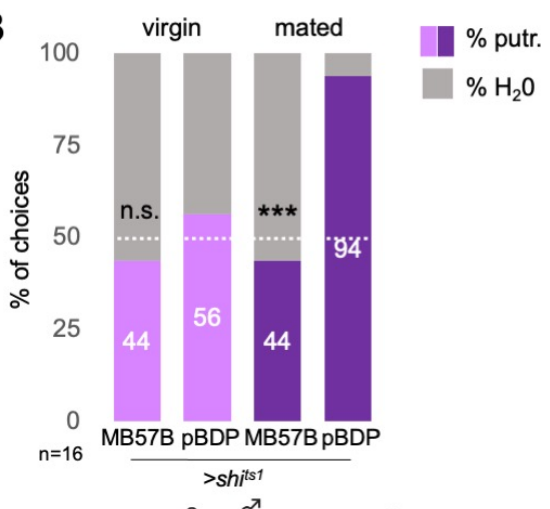

D
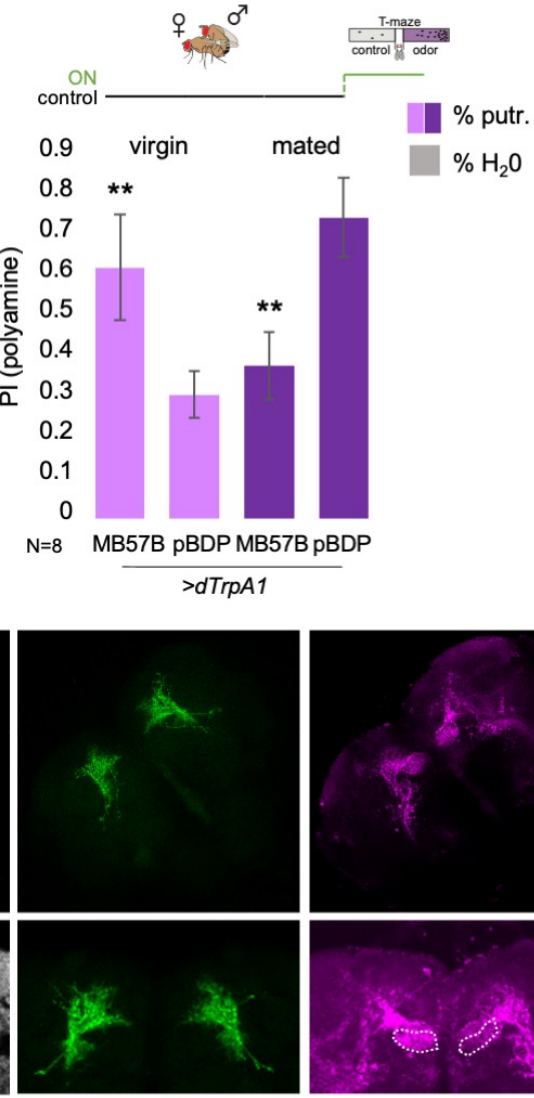

MB57B>mCD8GFP

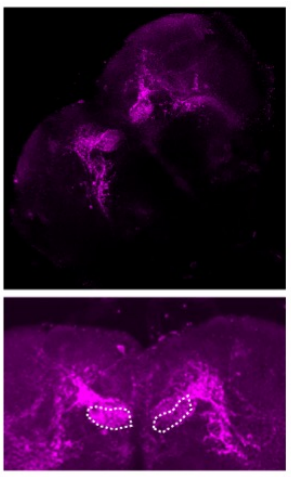

transTango >dsRed

Figure 4. Modulation of $\beta$ ' 1 mushroom body compartment changes female choice behavior.

(A) top, scheme of MBON- $\beta$ ' 1 and expression of line MB57B. bottom, scheme showing experimental paradigm. Neuronal output was blocked at the time of mating. (B) Inhibition of MBON- $\beta$ ' 1 output (MB57BGal4; $U A S-s h i^{t s I}$ ) during mating significantly reduced mated female preference for putrescine at the time of 
1065 significantly increased virgin female preference and decreased mated female preference for putrescine at the time of test, respectively. T-maze group assay. Bars depict preference index $(\mathrm{PI}) \pm \mathrm{SEM}$. Depicted p-values, unless indicated otherwise, refer to the difference between experimental and control group in virgins or mated females, respectively. Fisher's exact test. P-values: n.s.: $>0.1, *:<0.1, * *:<0.05, * * *:<0.001$. (E) Trans-TANGO experiment reveals a possible direct synaptic connection between MBON- $\beta$ ‘ 1 and MBON-

$1070 \beta$ '2. Grey, anti-Ncad stains neuropil, green, anti-GFP shows expression in MBON- $\beta$ ’1, magenta, anti-dsred stains expression of trans-TANGO mediated expression of dsRed in synaptically connected MBON- $\beta$ '2.

(E') Higher magnification of images shown in (E). 
A
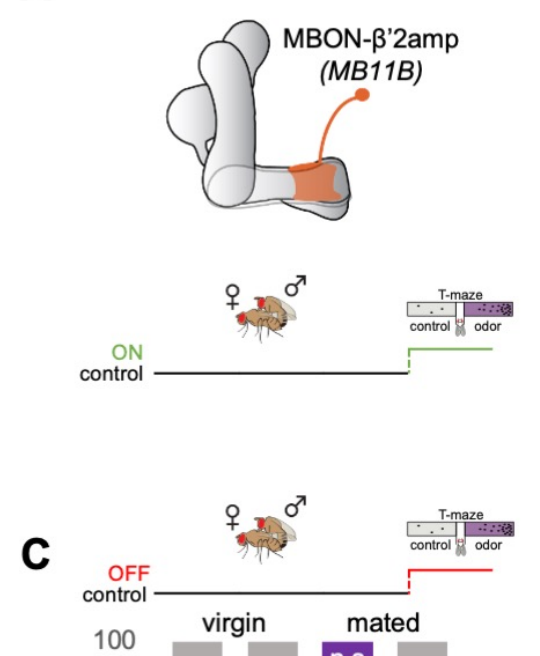

1075

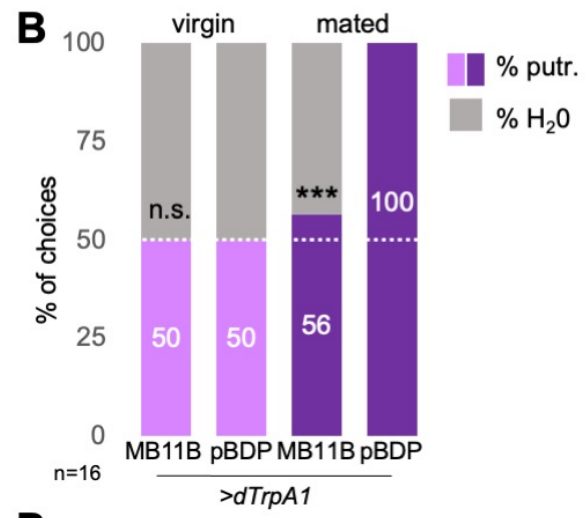

D

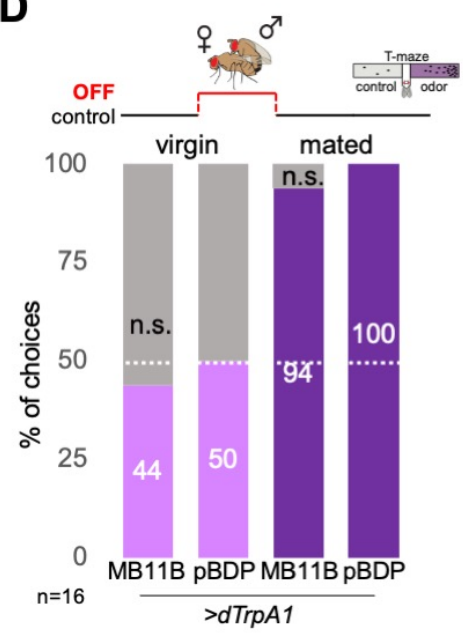

$\mathbf{F}$

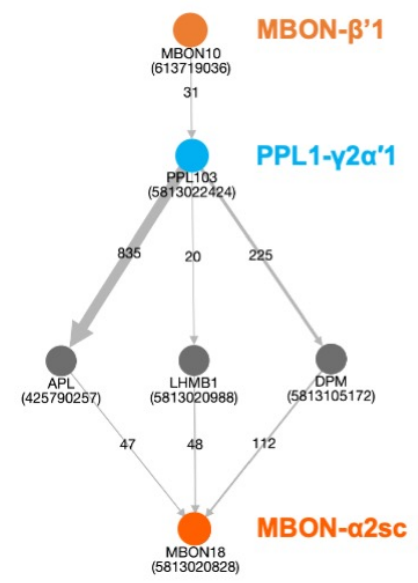

Figure 5. $\beta$ '2 output regulates degree of polyamine attraction.

(A) top, scheme of MBON- $\beta$ '2amp and expression of line MB11B. bottom, scheme showing experimental paradigm. Neurons were activated by dTrpA1 expression at the time of olfactory test. (B) Activation of MBON- $\beta$ '2 output (MB11B-Gal4;UAS-dTrpA1) during test significantly reduced mated female preference for putrescine. Single female preference for putrescine in T-maze assay. 50\% represents chance. (C) Inhibition of MBON- $\beta$ ' 2 output (MB11B-Gal4; UAS-shis ${ }^{t s I}$ ) during testing significantly increased virgin female preference for putrescine at the time of test. Single female preference for putrescine in T-maze assay. $50 \%$ represents chance. (D) Inhibition of MBON- $\beta$ '2 output (MB11B-Gal4;UAS-shi $\left.{ }^{\text {tsl }}\right)$ during mating or in lieu of mating had no effect on female preference. T-maze group assay. Bars depict preference index (PI) \pm SEM. (E) Activation MBON- $\beta$ '2 output (MB11B-Gal4;UAS-dTrpA1) instead of or during mating did not 
change female preference for putrescine at the time of test. Single female preference for putrescine in Tmaze assay. 50\% represents chance. Depicted p-values, unless indicated otherwise, refer to the difference between experimental and control group in virgins or mated females, respectively. Fisher's exact test. Pvalues: n.s.: $>0.1, *:<0.1, * *:<0.05, * * *:<0.001$. (F) EM-based connectome showing a pathway from MBON $\beta$ ' 1 to $\mathrm{MBON}-\alpha 2 \mathrm{sc}$.
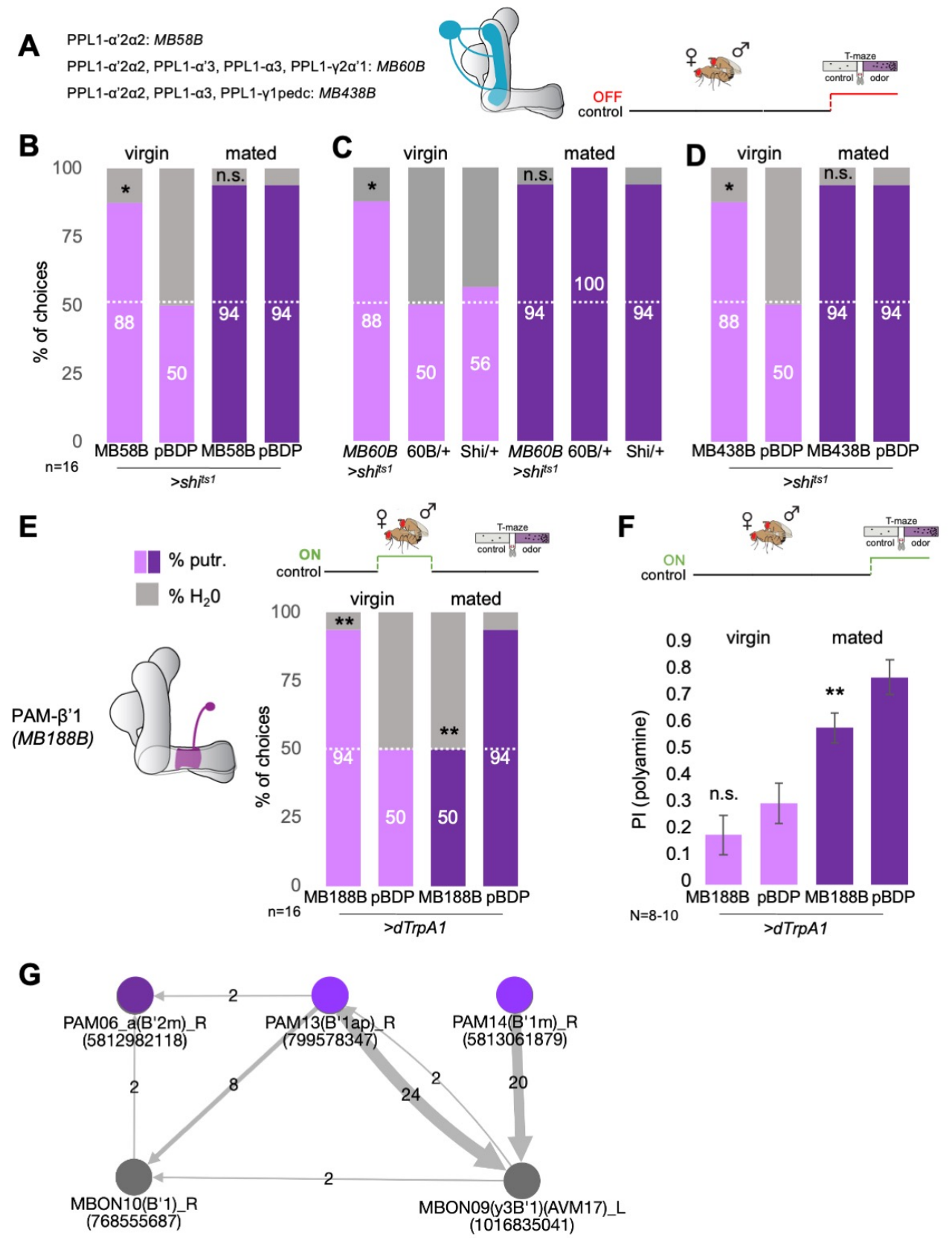

Figure 6. Specific dopaminergic neurons can mimic effect of mating.

1095 (A) left, scheme of PPL1 cluster of dopaminergic neurons and expression of three different transgenic lines. right, scheme showing experimental paradigm. Neuronal output was inhibited the time of olfactory test. (B) Inhibition of PPL1- $\alpha^{\prime} 2 \alpha 2$ output (MB58B-Gal4; UAS-shit ${ }^{\text {tsI }}$ ) during testing significantly increased virgin female preference for putrescine at the time of test. Single female preference for putrescine in T-maze assay. 
$50 \%$ represents chance. (C) Inhibition of PPL1- $\alpha^{\prime} 2 \alpha 2, \alpha 3, \gamma 2 \alpha^{\prime} 1$ output (MB60B-Gal4; UAS-shi $\left.{ }^{\text {tsl }}\right)$ during testing also significantly increased virgin female preference for putrescine at the time of test. Single female preference for putrescine in T-maze assay. 50\% represents chance. (D) Inhibition of PPL1- $\alpha^{\prime} 2 \alpha 2, \alpha 3$, $\gamma 1$ pedc output (MB438B-Gal4;UAS-shit $\left.{ }^{\text {tsI }}\right)$ during testing significantly increased virgin female preference for putrescine at the time of test. Single female preference for putrescine in T-maze assay. $50 \%$ represents chance. (E) Activation of PAM- $\beta$ '1 output (MB188B-Gal4;UAS-dTrpA1) instead of mating significantly

1105 increased virgin female preference for putrescine at the time of test. Vice versa, it significantly decreased the mated female's preference for the same at testing. Single female preference for putrescine in T-maze assay. 50\% represents chance. Fisher's exact test; P-values: n.s.: $>0.1, *:<0.1, * *:<0.05, * * *:<0.001$. (F) Activation of PAM- $\beta$ ' 1 output (MB188B-Gal4;UAS-dTrpA1) during olfactory preference test mildly, but significantly decreased mated female preference for putrescine at the time of test. T-maze group assay. Bars 1110 depict preference index $(\mathrm{PI}) \pm$ SEM. Depicted p-values, unless indicated otherwise, refer to the difference between experimental and control group in virgins or mated females, respectively. One-way Anova, Bonferroni-corrected. P-values: n.s.: $>0.1, *:<0.1, * *:<0.05, * * *:<0.001$. (G) EM-based connectome showing synaptic connections of PAM- $\beta$ ' 1 neurons. 

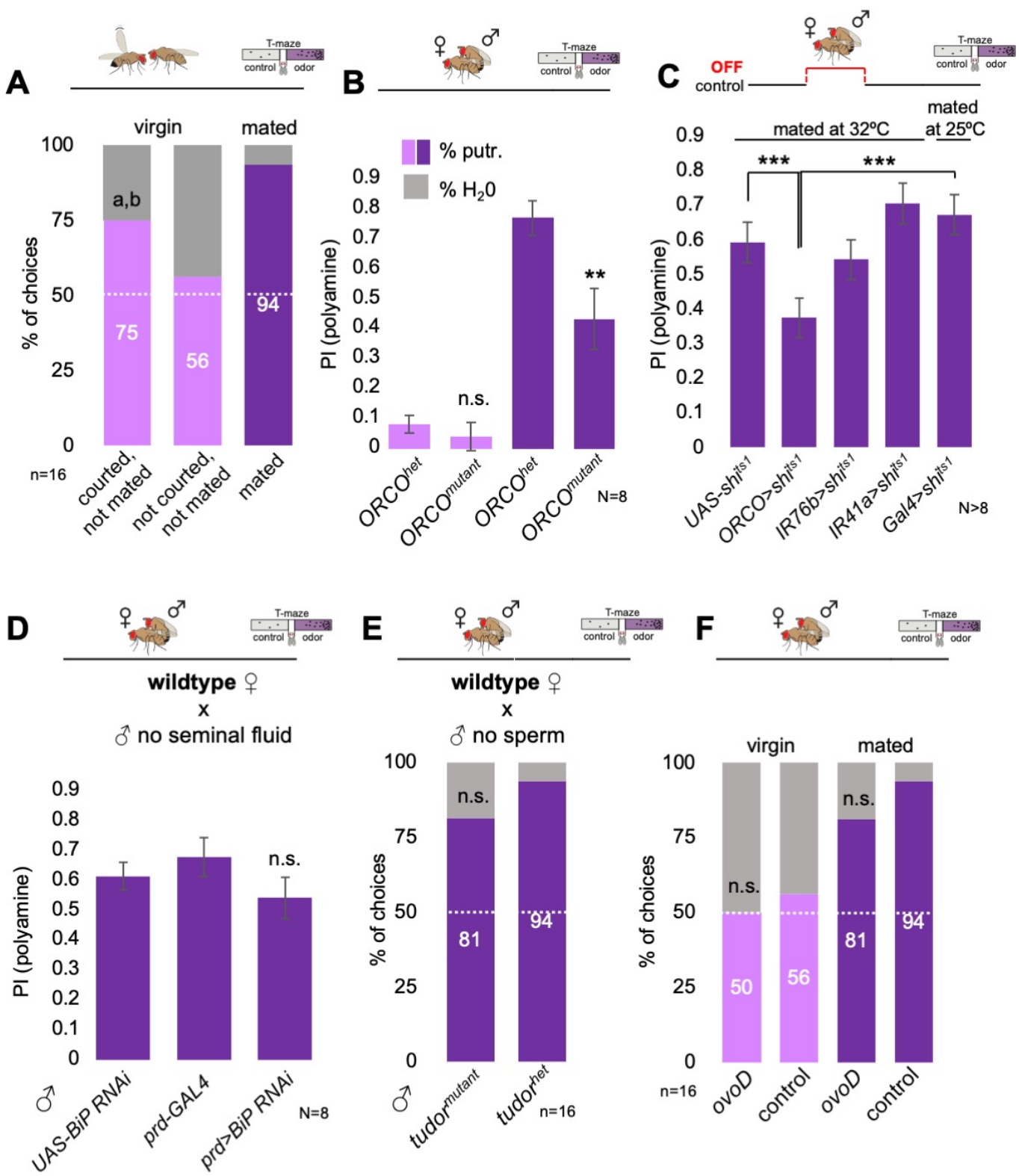

Figure 7. Courtship and odor detection contribute to lasting change in behavior.

(A) Courtship without mating induces higher preference in virgin females. Note that preference of courted, not mated virgins is not significantly different from mated females $(a, p>0.1)$ or virgin $(b, p>0.1)$. Single female preference for putrescine in T-maze assay. 50\% represents chance. (B) Females mutant for ORCO $\left(\right.$ ORCO $\left.{ }^{\text {mutant }}\right)$ showed a significantly reduced preference for polyamines during olfactory preference test compared to controls $\left(O R C O^{\text {het }}\right)$. Note that $\mathrm{ORCO}$ is not required for the detection of polyamine odor, but for the detection of pheromones and other food related odors. T-maze group assay. Bars depict preference index (PI) \pm SEM. Student-s t-test; P-values: n.s.: $>0.05,{ }^{*}:<0.05, * *:<0.01$ (C) T-maze group assay for females mated at restrictive temperature $\left(32^{\circ} \mathrm{C}\right)$ and tested at permissive temperature for their polyamine preference $\left(25^{\circ} \mathrm{C}\right)$. In addition, flies of the same genotypes (pooled) were mated and tested at $25^{\circ} \mathrm{C}$ as a control. Note that synaptic inhibition of ORCO-expressing OSNs during mating significantly reduced the females' attraction to putrescine odor. T-maze group assay. Bars depict preference index $(\mathrm{PI}) \pm \mathrm{SEM}$. Student-s t-test; P-values: n.s.: $>0.05, *:<0.05, * *:<0.01$ (D) Seminal fluid is not required to induce postmating polyamine preference. Wildtype CantonS females were mated with males without seminal fluid 
1130 (prd-Gal4;UAS-BiP RNAi) or with control males (prd-Gal4/+ or UAS-BiP RNAi/+). T-maze group assay. Bars depict preference index (PI) \pm SEM. Student-s t-test; P-values: n.s.: $>0.05, *:<0.05, * *:<0.01$ (E) Sperm is not essential for postmating polyamine preference induction. Wildtype CantonS females were mated with sterile males unable to produce sperm $\left(t u d o r^{\text {mutant }}\right)$ or with control males $\left(t u d^{\text {het }}\right)$. Single female preference for putrescine in T-maze assay. 50\% represents chance. (F) Egg production does not drive

1135 female's increased preference for polyamines. Mated females unable to produce eggs (ovoD) behaved no different from control females producing eggs. Single female preference for putrescine in T-maze assay. $50 \%$ represents chance. Depicted p-values, unless indicated otherwise, refer to the difference between experimental and control group in virgins or mated females, respectively. Fisher's exact test. P-values: n.s.: $>0.1, *:<0.1, * *:<0.05, * * *:<0.001$.

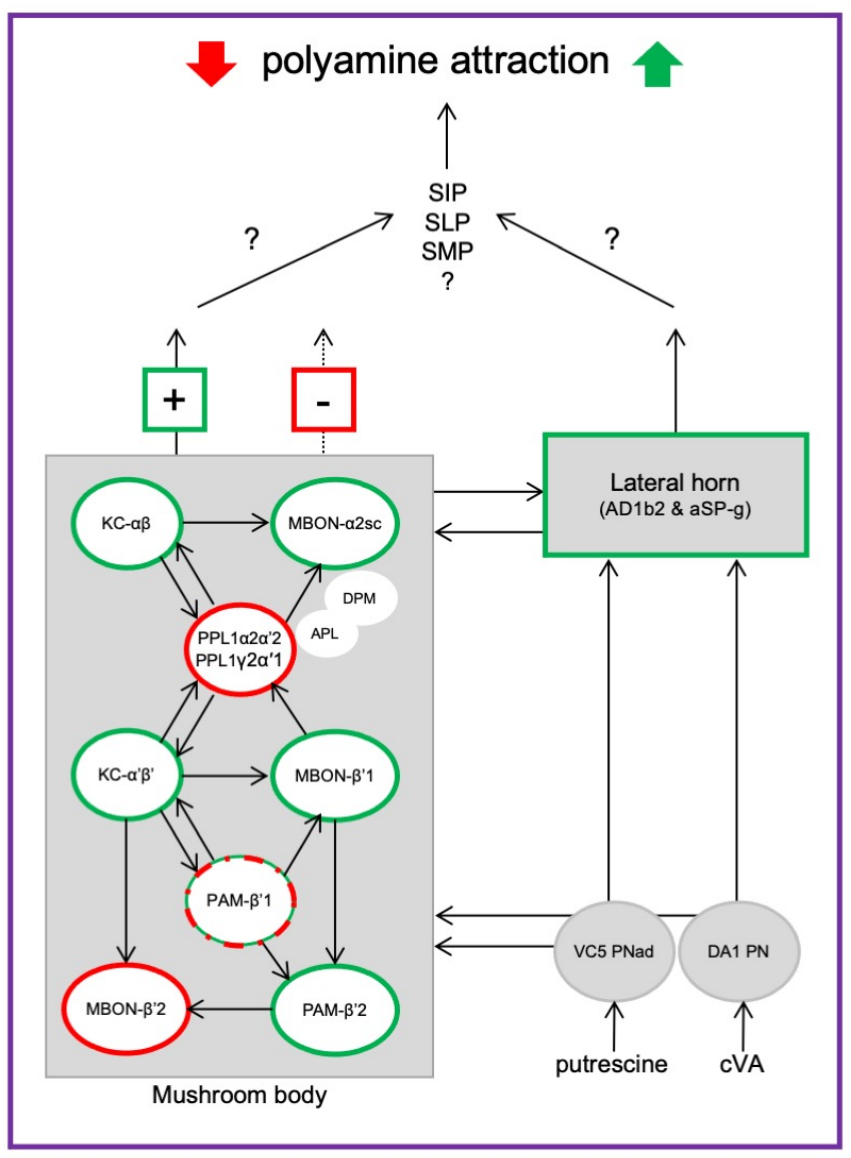

Figure 8. Polyamine attraction relies on multiple pathways

Working model summarizing behavioral data. Polyamine odor (putrescine) activates the VC5 glomerulus in 1145 the antennal lobe. VC5 projections neurons (PNs) send odor information to mushroom body (MB) and lateral horn (LH). Red circled neurons/brain regions highlight parts of the circuit that are involved in reducing polyamine attraction. Green circled neurons/brain regions highlight parts of the circuit that are involved in increasing polyamine attraction. For instance, activation of MBON- $\beta$ ' 2 and PPL1-DANs at test inhibits preference for putrescine, while PAM- $\beta$ ' 1 activation instead of/during mating increases polyamine attraction in virgins and decreases attraction in mated females, respectively (dotted red-green circle). MBON- $\beta$ ' 1 output is required during mating and activation is sufficient to induce polyamine attraction in virgins. However, activation in mated flies during testing reduces the attraction suggesting a more 
bioRxiv preprint doi: https://doi.org/10.1101/2021.07.24.453623; this version posted July 25, 2021. The copyright holder for this preprint (which

was not certified by peer review) is the author/funder, who has granted bioRxiv a license to display the preprint in perpetuity. It is made available under aCC-BY-NC-ND 4.0 International license.

1160

complicated relationship between MBON activity and preference behavior, possibly due to the many recurrent connections observed in the circuit. We suggest that the mating induced increase in polyamine

1155 attraction occurs through the modulation of LH and MB outputs, which both activate downstream neurons in the superior protocerebrum. We hypothesize that pheromones, including the LHON aSP-g, comprise a part of the mating-related signals that induce increased polyamine attraction. 